\title{
9. CLAY MINERAL DISTRIBUTION AND SIGNIFICANCE IN QUATERNARY SEDIMENTS OF THE AMAZON FAN ${ }^{1}$
}

\author{
P. Debrabant, ${ }^{2}$ M. Lopez, ${ }^{2}$ and H. Chamley ${ }^{2}$
}

\begin{abstract}
The clay mineral assemblages of samples from the Amazon Fan were investigated in middle Pleistocene to Holocene sediments from 16 Ocean Drilling Program Leg 155 sites and were placed in lithologic successions to study continental evolution and transport conditions. The sediments sampled along a transect parallel to the main channel reflect moderate differential settling processes, whereas those studied along a transect roughly parallel to the coast clearly suggest winnowing effects related to the North Brazil Current. High-resolution analyses of Holocene deposits reflect the dominant influence of downstream soil erosion relative to supply from the Andean range and the progressive increase of soil-derived relative to rock-derived outputs. The middle Pleistocene to uppermost Pleistocene and Holocene successions are marked by a constantly warm climatic background and by noticeable modifications in the rock-derived (illite, chlorite) and soil-derived (smectite, kaolinite) products. These products successively reflect sea-level changes, tectonic rejuvenation in the Andean range, regressive erosion, and rainfall increase.
\end{abstract}

\section{INTRODUCTION}

The Amazon Fan is one of the world's largest modern submarine fan systems. It extends $700 \mathrm{~km}$ seaward of the continental shelf of Brazil on an area of $375,000 \mathrm{~km}^{2}$ in a water depth of $1000-4300 \mathrm{~m}$ (Fig. 1). It represents a typical passive margin deep-sea fan (Stow et al., 1985) developed since major Andean uplift in the late Miocene caused the connection of the Amazon Basin with the Atlantic Ocean and the drowning of the carbonate platforms (Hoorn, 1994; Hoorn et al., 1995). The present high sea-level stand prevents the Amazon River from crossing the submerged shelf (Nittrouer et al., 1991; Geyer et al., 1991), and the late Pleistocene fan deposits are covered by a thin hemipelagic drape. During glacial low sea-level stands, the river discharged directly into the submarine canyon which crossed the continental slope and led to a large sinuous channel-levee system. This submarine topography is fairly well known due to extensive survey, swath-mapping bathymetry (SeaBeam) and deep-water side-scan sonar (GLORIA; Damuth et al., 1983; Manley and Flood, 1988). By contrast, the lithology and age of the late Neogene to Quaternary sediments that make up these systems, as well as the relationships between these systems and sea-level changes, were, until recently, rather poorly defined because of the lack of drilling cores.

Leg 155 of the Ocean Drilling Program (ODP) bridged this gap by drilling 17 sites (Fig. 1). The recovery of $4000 \mathrm{~m}$ of sediment series permitted the shipboard scientists to meet four main objectives: (1) establish the relationships between the development of the fan deposits, sea-level fluctuations, climate change, and possible tectonic activity; (2) determine the sediment lithologies characteristic of distinct acoustic facies and flow processes; (3) increase the precision of the stratigraphic record; and (4) understand the nature and origin of the early diagenesis of organic carbon present in different fan units.

The present work approaches the first objective by using the clay mineral record of selected cores to evaluate the respective influence of the land climate, sea-level/base-level changes, ocean circulation, and tectonic activity during fan growth. The clay mineral assemblag-

${ }^{1}$ Flood, R.D., Piper, D.J.W., Klaus, A., and Peterson, L.C. (Eds.), 1997. Proc. ODP, Sci. Results, 155: College Station, TX (Ocean Drilling Program).

${ }^{2}$ Sédimentologie et Géodynamique, URA 719 du CNRS, Université de Lille, 59655 Villenueuve d'Ascq, France. es of late Cenozoic sediments from the Atlantic Ocean are almost exclusively of a detrital origin (Biscaye, 1965; Robert, 1982; Chamley, 1989), even though clay minerals may locally form in shallow-water environments (Michalopoulos and Aller, 1995). These terrigenous materials, which are dominantly eroded from soil and weathering profile formations and secondarily from rocky substrates, are able to express the paleoenvironmental changes that have characterized the continental climate by developing various pedogenetic conditions, the movements of marine water masses carrying these fine detrital particles, and tectonic uplifts favoring the direct erosion of soils (e.g., Chamley, 1989; Weaver, 1989; Fig. 2).

The mud-rich deposits and high sedimentation rates that often characterize the deep-sea fans are particularly suitable for high-resolution paleoenvironmental reconstitutions. For instance, previous investigations performed in Neogene to Quaternary deposits from Ganges and Indus deep-sea fans showed that the presence and the qualitative and quantitative variations of clay mineral assemblages express the successive steps of tectonic, climatic, and eustatic events that controlled the building of the fan (Bouquillon, 1987; Bouquillon and Debrabant, 1987; Debrabant et al., 1991; Fagel, 1994). High-resolution studies on distal sediments from these fans proved the ability of clay minerals to record the periodic alternations of climate (Fagel et al., 1992; 1994).

The present study focuses on two topics linked to the late Quaternary history of the Amazon Fan: (1) the horizontal and vertical distribution of clay minerals in Holocene sediments and at the last glacialinterglacial transition; and (2) the clay mineral record of superimposed channel-levee systems illustrated by the continuous clay mineral record of Hole 931B.

\section{MATERIAL AND METHODS}

Seventeen holes corresponding to 16 sites from Leg 155 were sampled (Table 1), first along the present Amazon Channel over a distance of $260 \mathrm{~km}$ (Sites 937-946) and second on an east-west transect over a distance of $280 \mathrm{~km}$ (Sites 931-942). The clay mineralogy of the detrital sediments was investigated from three sampling groups. First, a general determination of the clay mineral composition and diversity was performed from 16 sites on 33 near-surface 
Figure 1. The Amazon Fan and location of Leg 155 drilling sites. Modified from Damuth et al., 1988, and Manley and Flood, 1988.

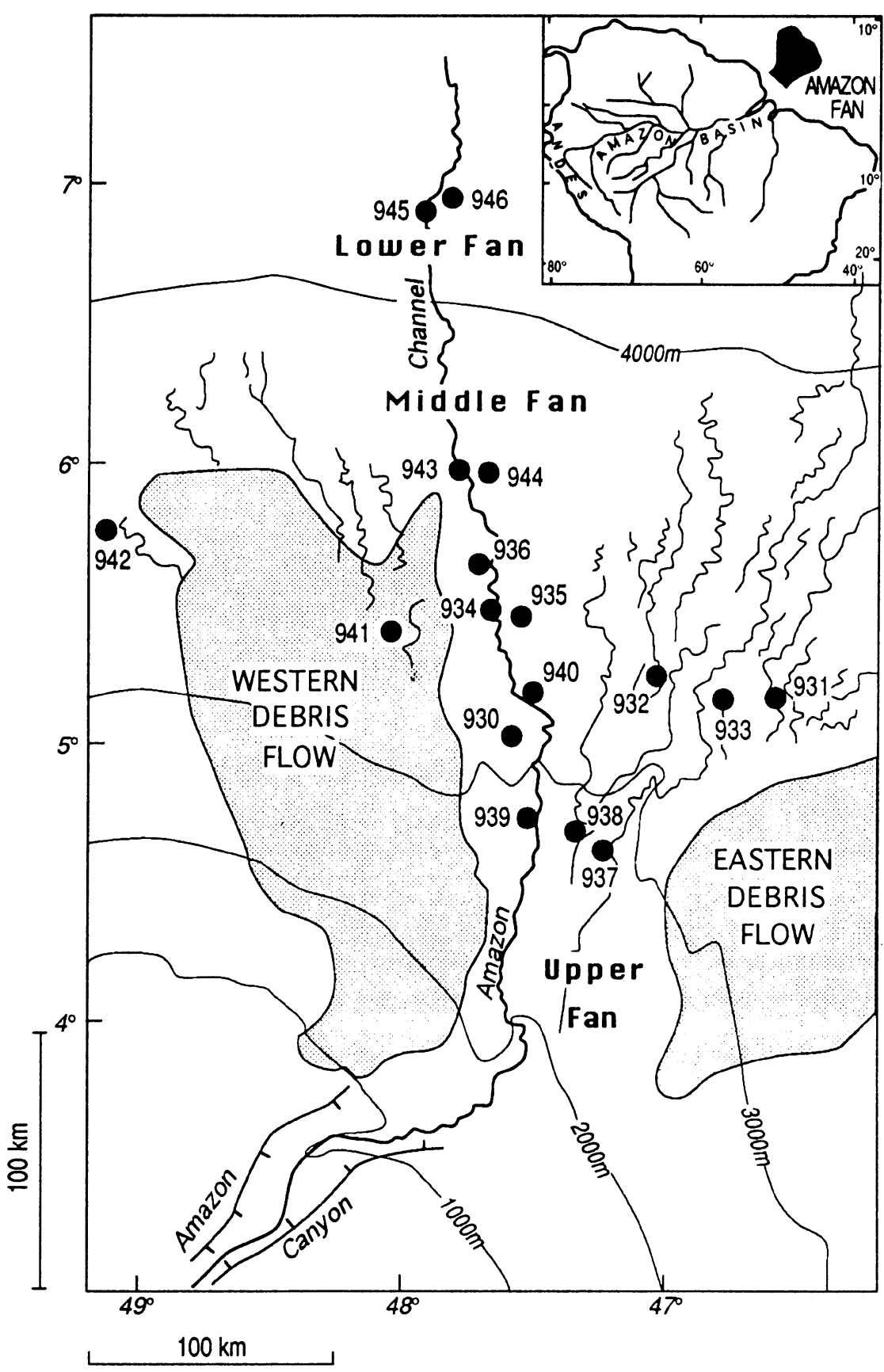

sediments sampled between 0.1 and 5.5 meters below seafloor (mbsf; uppermost Pleistocene to Holocene; Table 2). Second, a high-resolution study was conducted on 40 samples $7-20 \mathrm{~cm}$ distant from each other in the Holocene sediments of Site 934 (Table 3). Third, the clay mineral stratigraphy at Hole 931B was determined from 73 samples located between 0.34 and 415.07 mbsf (Table 4).

The clay-mineral data were obtained using X-ray diffraction (XRD) on the $<2-\mu \mathrm{m}$ size sedimentary fraction. Samples disintegrated in deionized water were decalcified with $0.2 \mathrm{~N} \mathrm{HCl}$ as quickly as possible and then buffered; the $<2-\mu \mathrm{m}$ size fraction was separated by settling. Oriented mounts were then prepared on glass slides and scanned three times $\left(\mathrm{Cu} \mathrm{K} \mathrm{K}_{\alpha}\right.$ radiation) from $2.5^{\circ}$ to $28.5^{\circ} 2 \theta$ on untreated samples and on glycolated samples, and from $2.5^{\circ}$ to $14.5^{\circ} 2 \theta$ on samples heated for $2 \mathrm{hr}$ at $490^{\circ} \mathrm{C}$. Analytical techniques, determi- nation, and quantitative estimations of clay minerals are detailed in Debrabant et al. (1984) and Holtzapffel (1985). Analytical uncertainties in the abundance of chlorite, illite, smectite, kaolinite, and random mixed layers are estimated at $\pm 3 \%$ for abundances above $20 \%$, $\pm 2 \%$ for abundances between $20 \%$ and $5 \%$, and $\pm 1 \%$ for abundances below $5 \%$. Trace amounts are reported when the abundance of a clay species is hardly measurable and represents less than $2 \%$ of the total clay minerals. The reproducibility of the quantitative estimates has been checked by successive XDR runs of a given sample. X-ray traces also indicate the presence of nonphyllosilicate minerals (especially quartz and feldspar), whose abundances cannot be quantified using this technique.

The random mixed-layer clay minerals that represent intermediate structures between two single clay species (e.g., illite and smectite; 

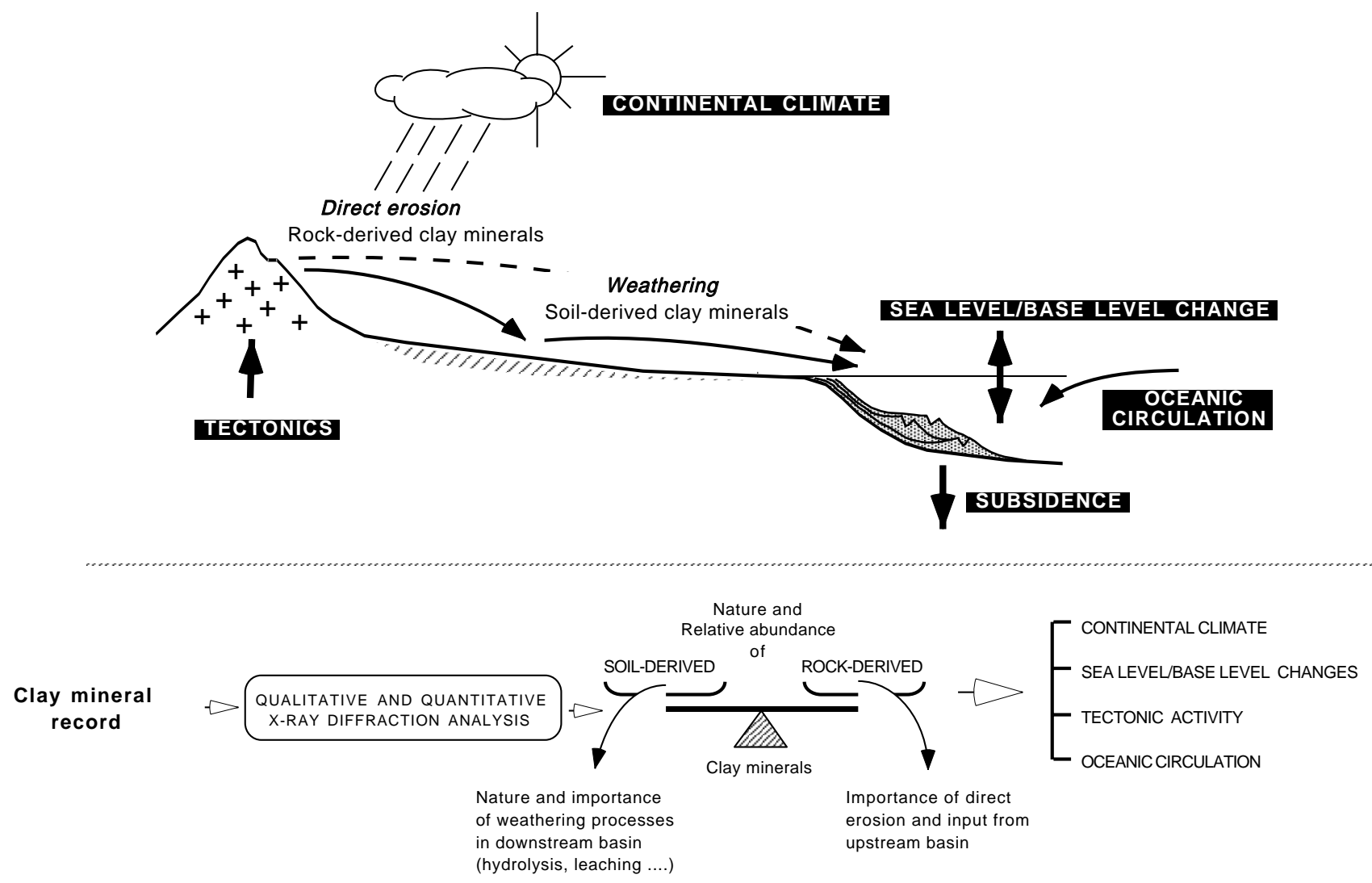

Figure 2. Schematic sketch showing how clay minerals can help clarify the history of the Amazon Fan.

Table 1. Location of Leg 155 drilling sites investigated.

\begin{tabular}{clrrr}
\hline Hole & \multicolumn{1}{c}{ Position } & $\begin{array}{c}\text { Water } \\
\text { depth } \\
(\mathrm{m})\end{array}$ & $\begin{array}{c}\text { Penetration } \\
(\mathrm{m})\end{array}$ & $\begin{array}{c}\text { Depth interval } \\
\text { investigated } \\
(\mathrm{mbsf})\end{array}$ \\
\hline $930 \mathrm{~B}$ & $5^{\circ} 0.894^{\prime} \mathrm{N}, 47^{\circ} 35.743^{\prime} \mathrm{W}$ & 3143.6 & 225.30 & $0.15-2.30$ \\
$931 \mathrm{~A}$ & $5^{\circ} 8.511^{\prime} \mathrm{N}, 46^{\circ} 37.983^{\prime} \mathrm{W}$ & 3472.7 & 53.60 & $0.01-3.99$ \\
$931 \mathrm{~B}$ & $5^{\circ} 8.521^{\prime} \mathrm{N}, 46^{\circ} 37.984^{\prime} \mathrm{W}$ & 3475.9 & 421.30 & $0.34-415.07$ \\
$933 \mathrm{~A}$ & $5^{\circ} 5.801^{\prime} \mathrm{N}, 46^{\circ} 48.738^{\prime} \mathrm{W}$ & 3365.9 & 254.20 & $0.29-3.64$ \\
$934 \mathrm{~A}$ & $5^{\circ} 29.047^{\prime} \mathrm{N}, 47^{\circ} 40.857^{\prime} \mathrm{W}$ & 3421.7 & 108.80 & $0.13-4.07$ \\
$935 \mathrm{~A}$ & $5^{\circ} 25.612^{\prime} \mathrm{N}, 47^{\circ} 33.893^{\prime} \mathrm{W}$ & 3485.5 & 372.60 & $0.24-2.05$ \\
$936 \mathrm{~A}$ & $5^{\circ} 37.936^{\prime} \mathrm{N}, 47^{\circ} 44.134^{\prime} \mathrm{W}$ & 3575.0 & 433.80 & $0.30-1.99$ \\
$937 \mathrm{~B}$ & $4^{\circ} 35.749^{\prime} \mathrm{N}, 47^{\circ} 12.449^{\prime} \mathrm{W}$ & 2759.9 & 180.30 & $0.25-0.75$ \\
$938 \mathrm{~A}$ & $4^{\circ} 39.504^{\prime} \mathrm{N}, 47^{\circ} 18.740^{\prime} \mathrm{W}$ & 2804.1 & 310.20 & $0.41-0.98$ \\
$939 \mathrm{~B}$ & $4^{\circ} 43.305^{\prime} \mathrm{N}, 47^{\circ} 29.940^{\prime} \mathrm{W}$ & 2792.3 & 99.40 & $0.15-2.30$ \\
$940 \mathrm{~A}$ & $5^{\circ} 8.596^{\prime} \mathrm{N}, 47^{\circ} 31.728^{\prime} \mathrm{W}$ & 3191.4 & 248.60 & $0.08-2.30$ \\
$941 \mathrm{~A}$ & $5^{\circ} 22.384^{\prime} \mathrm{N}, 48^{\circ} 1.723^{\prime} \mathrm{W}$ & 3380.9 & 177.90 & $0.00-2.10$ \\
$942 \mathrm{~A}$ & $5^{\circ} 44.546^{\prime} \mathrm{N}, 49^{\circ} 5.464^{\prime} \mathrm{W}$ & 3346.3 & 177.60 & $0.53-0.96$ \\
$943 \mathrm{~A}$ & $5^{\circ} 56.808^{\prime} \mathrm{N}, 47^{\circ} 46.831^{\prime} \mathrm{W}$ & 3738.5 & 106.30 & $0.18-3.24$ \\
$944 \mathrm{~A}$ & $5^{\circ} 56.335^{\prime} \mathrm{N}, 47^{\circ} 45.469^{\prime} \mathrm{W}$ & 3701.3 & 384.20 & $0.14-2.43$ \\
$945 \mathrm{~A}$ & $6^{\circ} 57.019^{\prime} \mathrm{N}, 47^{\circ} 55.732^{\prime} \mathrm{W}$ & 4136.1 & 75.50 & $0.19-5.54$ \\
$946 \mathrm{~A}$ & $6^{\circ} 56.977^{\prime} \mathrm{N}, 47^{\circ} 55.161^{\prime} \mathrm{W}$ & 4100.5 & 275.00 & $0.22-3.77$ \\
\hline
\end{tabular}

Brindley and Brown, 1980; Moore and Reynolds, 1989) are labeled as follows (Lucas, 1962): (10-14s) illite-smectite; (10-14v) illitevermiculite; and $(14 \mathrm{c}-14 \mathrm{~s})$ chlorite-smectite.

Additional information is provided by five indices calculated from the ratio of peak heights measured on the X-ray trace of the glycolated samples: (1) kaolinite + chlorite/illite $7 \AA / 10 \AA$ peak height ratio; (2) smectite/illite $17 \AA / 10 \AA$ peak height ratio; (3) kaolinite + chlorite/smectite $7 \AA / 17 \AA$ peak height ratio; (4) kaolinite/chlorite $3.57 \AA / 3.54 \AA$ A peak height ratio; and (5) smectite crystallinity $17 \AA$ peak height/width, the width being measured at half-height. This index was chosen for its easy determination and its relative indepen- dence of the smectite concentrations (Tables 3, 4). Non-clay minerals present in the clay-size-fraction $(<2-\mu \mathrm{m}$ particles) mainly consist of quartz and feldspars, the abundance of which has been estimated from peak height as either rare (+) or common (++).

\section{SPACE AND TIME DISTRIBUTION OF CLAY MINERALS AT THE LAST GLACIAL- INTERGLACIAL TRANSITION}

The study of clay-mineral distribution during the last sea-level rise comprises two complementary approaches: (1) a comparative analysis at each site of two samples respectively located in late Pleistocene and Holocene sediments to clarify the mineral distribution during transitional time and highstand; and (2) a high-resolution study by closely spaced sampling of a well-preserved transitional to highstand record.

\section{Surface Distribution in Holocene Sediments} Stratigraphy and Sedimentary History

The first meters of the sediment record encompass the last sealevel rise and correspond roughly to the $\mathrm{Z}$ ecostratigraphic Ericson Zone (Ericson et al., 1961; Ericson and Wollin, 1968), which is approximately equivalent to isotopic Stage 1 . The Z/Y Ericson boundary dated at $11 \mathrm{ka}$ is supposed to be coeval with the Holocene/late Pleistocene boundary (Shipboard Scientific Party, 1995a).

The Holocene lithology is very constant throughout the fan. It consists of two distinct units in which the sampling for clay mineral analysis was done. Unit I consists of $0.24-1.18 \mathrm{~m}$ of brown to yellowish brown, moderately burrowed calcareous clay containing 
Table 2. Clay mineral composition of Amazon Fan surface sediments (\%)

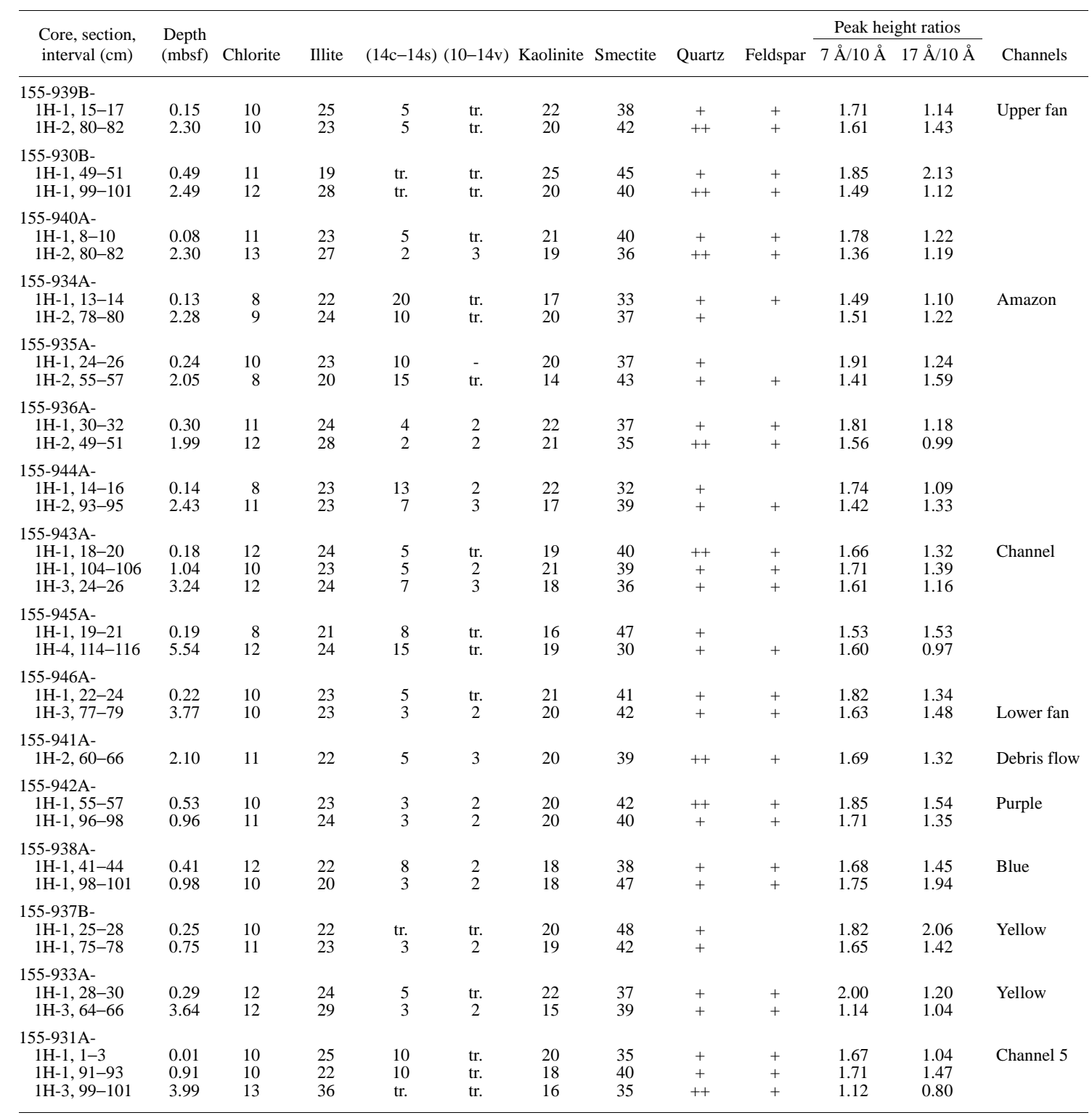

Note: $+=$ rare $++=$ common; tr. $=$ trace.

abundant foraminifer and nannofossil oozes. From base to top, the carbonate fraction increases from $\sim 10 \%$ to $\sim 30 \%-40 \%$. This increase is often expressed by a gradational color change from grayish brown at the base to yellowish brown at the top. The base of this unit contains one to five millimeter- to centimeter-thick, diagenetic, rust-colored iron-rich crusts. The crusts have been analyzed and correlated throughout the Amazon Fan and adjacent Guyana Basin (Damuth, 1977). They probably correspond to a redox boundary. Unit I is interpreted as hemipelagic mud.

Unit II comprises in its uppermost part defined as Subunit IIA, dark gray nannofossil- and foraminifer-bearing clay that displays a transitional contact with Unit I. This facies is characterized by extensive color mottling that is attributed to heavy bioturbation and burrowing filled by pervasive black hydrotroilite. Halo-burrows are especially abundant. This facies grades downward in the late Pleistocene section to dark gray banded silty clay (Subunit IIB). Unit II is interpreted as clayey mud resulting from distal terrigenous input. It marks the last phase of turbiditic activity in the Amazon Fan during the sea-level rise. Unit I and Subunit IIA were sampled at each site.

\section{Clay Mineralogy}

\section{General data}

The clay mineral distribution of recent sediments in the Amazon Fan displays a fairly constant composition. Smectite constitutes the more abundant species (30\% to $50 \%$ of the clay minerals) and is associated with illite $(20 \%-35 \%)$, kaolinite $(15 \%-25 \%)$, chlorite $(8 \%-$ $12 \%$ ), and random mixed layers (trace amounts to $20 \%$, chloritesmectite being more frequent than illite-vermiculite). Quartz is rare to common, and feldspars occur as trace amounts; both associated non-clay minerals are ubiquitous. The X-ray traces (Fig. 3) are very similar to those obtained by Patchineelam (1993). Notice that (1) smectite is moderately well crystalline and probably includes expandable layers rich in random illite-smectite mixed layers, (2) chlo- 
Table 3. Clay mineral composition (\%) of the Amazon Fan sediments in the uppermost cores of Hole 934A.

\begin{tabular}{|c|c|c|c|c|c|c|c|c|c|c|c|c|c|c|c|c|}
\hline \multirow{2}{*}{$\begin{array}{l}\text { Core, section, } \\
\text { interval }(\mathrm{cm})\end{array}$} & \multirow{2}{*}{$\begin{array}{l}\text { Depth } \\
\text { (mbsf) }\end{array}$} & \multirow[b]{2}{*}{ Lithology } & \multirow[b]{2}{*}{ Chlorite } & \multirow[b]{2}{*}{ Illite } & \multirow[b]{2}{*}{$(14 c-14 s)$} & \multirow[b]{2}{*}{$10-14 v)$} & \multirow[b]{2}{*}{$(14 v-14 s)$} & \multirow[b]{2}{*}{ Kaolinite } & \multirow[b]{2}{*}{ Smectite } & \multicolumn{4}{|c|}{ Peak height ratios } & \multirow{2}{*}{$\begin{array}{l}\text { Smectite } \\
\text { crystallinity }\end{array}$} & \multirow[b]{2}{*}{ Quartz } & \multirow[b]{2}{*}{ Feldspar } \\
\hline & & & & & & & & & & $7 \AA ̊ \AA 10 \AA$ & $17 \AA / 10 \AA$ & $7 \AA ̊ \AA / 17 \AA$ & $3.57 \AA / 3.54 \AA$ & & & \\
\hline \multicolumn{17}{|l|}{$155-934 \mathrm{~A}-$} \\
\hline $1 \mathrm{H}-1,13-14$ & 0.13 & Brown n.f.ooze & 8 & 22 & 20 & tr. & - & 17 & 33 & 1.49 & 1.10 & 1.36 & 2.80 & 4.67 & + & + \\
\hline $1 \mathrm{H}-1,16-28$ & 0.26 & ? & 7 & 22 & 5 & 18 & 30 & 18 & - & 1.52 & & & 3.31 & & + & + \\
\hline $1 \mathrm{H}-1,12-33$ & 0.32 & Brown n.f.ooze & 10 & 26 & tr. & tr. & - & 25 & 39 & 1.76 & 1.19 & 1.48 & 3.92 & 5.52 & + & \\
\hline $1 \mathrm{H}-1,18-49$ & 0.48 & Iron crust I & 9 & 22 & 10 & 5 & - & 16 & 48 & 1.37 & 1.58 & 0.87 & 2.92 & 4.44 & + & \\
\hline $1 \mathrm{H}-1,55-56$ & 0.55 & Dark gray clay & 11 & 26 & 5 & tr. & - & 20 & 38 & 1.56 & 1.17 & 1.32 & 2.55 & 5.68 & + & \\
\hline $1 \mathrm{H}-1,74-75$ & 0.74 & Light gray clay & 10 & 19 & 5 & 15 & - & 19 & 32 & 1.89 & 1.39 & 1.35 & 2.76 & 5.86 & + & \\
\hline $1 \mathrm{H}-1,86-87$ & 0.86 & Iron crust II & 8 & 18 & 8 & 2 & - & 16 & 48 & 1.57 & 1.91 & 0.82 & 3.50 & 4.47 & + & \\
\hline $1 \mathrm{H}-1,107-108$ & 1.07 & Clay & 10 & 24 & 8 & 2 & $\overline{0}$ & 20 & 36 & 1.65 & 1.18 & 1.39 & 2.62 & 5.13 & + & \\
\hline $1 \mathrm{H}-1,132-134$ & 1.32 & Dark clay & 8 & 20 & 5 & 18 & 32 & 17 & - & 1.59 & - & - & 3.00 & - & + & + \\
\hline \multicolumn{17}{|c|}{ High-resolution sequence } \\
\hline $1 \mathrm{H}-2,2-3$ & 1.52 & Black clay & 9 & 26 & 3 & 2 & - & 20 & 40 & 1.50 & 1.27 & 1.18 & 3.14 & 6.08 & + & + \\
\hline $1 \mathrm{H}-2,6-7$ & 1.56 & Light clay & 12 & 27 & tr. & tr. & - & 22 & 39 & 1.65 & 1.15 & 1.44 & 2.52 & 6.37 & + & + \\
\hline $1 \mathrm{H}-2,14-15$ & 1.64 & Black clay & 11 & 26 & 3 & 2 & - & 19 & 39 & 1.51 & 1.14 & 1.32 & 2.53 & 4.87 & + & T \\
\hline $1 \mathrm{H}-2,22-23$ & 1.72 & Light clay & 11 & 25 & 3 & 2 & - & 19 & 40 & 1.59 & 1.29 & 1.24 & 2.26 & 5.85 & + & \\
\hline $1 \mathrm{H}-2,28-29$ & 1.78 & Light clay & 11 & 27 & 5 & tr. & - & 22 & 35 & 1.67 & 1.07 & 1.56 & 2.52 & 6.14 & + & \\
\hline $1 \mathrm{H}-2,40-41$ & 1.90 & Black clay & 13 & 27 & 5 & tr. & - & 17 & 38 & 1.49 & 1.10 & 1.35 & 1.64 & 5.92 & + & \\
\hline $1 \mathrm{H}-2,46-47$ & 1.96 & Light clay & 12 & 23 & 8 & 2 & - & 19 & 36 & 1.70 & 1.25 & 1.37 & 2.18 & 5.85 & + & + \\
\hline $1 \mathrm{H}-2,58-59$ & 2.08 & Black clay & 12 & 26 & 5 & tr. & - & 20 & 37 & 1.53 & 1.11 & 1.42 & 2.26 & 6.23 & + & \\
\hline $1 \mathrm{H}-2,61-62$ & 2.11 & Light clay & 12 & 27 & 5 & tr. & - & 21 & 35 & 1.62 & 1.03 & 1.58 & 2.21 & 5.63 & + & \\
\hline $1 \mathrm{H}-2,71-72$ & 2.21 & Light clay & 12 & 26 & 8 & 2 & - & 16 & 36 & 1.36 & 1.08 & 1.27 & 2.00 & 5.47 & + & \\
\hline $1 \mathrm{H}-2,75-76$ & 2.25 & Black clay & 10 & 26 & $\begin{array}{l}0 \\
10\end{array}$ & tr. & $\bar{z}$ & $\begin{array}{l}10 \\
18\end{array}$ & 36 & $\begin{array}{l}1.30 \\
1.32\end{array}$ & $\begin{array}{l}1.11 \\
1.11\end{array}$ & 1.19 & 2.47 & 6.08 & $\begin{array}{l}+ \\
+\end{array}$ & \\
\hline $1 \mathrm{H}-2,78-80$ & 2.28 & ? & 9 & 24 & 10 & $\begin{array}{l}\text { U. } \\
\text { tr. }\end{array}$ & - & $\begin{array}{l}10 \\
20\end{array}$ & $\begin{array}{l}50 \\
37\end{array}$ & $\begin{array}{l}1.52 \\
1.51\end{array}$ & $\begin{array}{l}1.11 \\
1.22\end{array}$ & $\begin{array}{l}1.19 \\
1.24\end{array}$ & 2.87 & $\begin{array}{l}0.00 \\
5.67\end{array}$ & $\begin{array}{l}+ \\
+\end{array}$ & \\
\hline $1 \mathrm{H}-2,87-88$ & 2.37 & Light clay & 10 & 23 & 8 & 2 & - & 16 & 41 & 1.47 & 1.39 & 1.06 & 2.35 & 5.91 & + & \\
\hline $1 \mathrm{H}-2,95-96$ & 2.45 & Black clay & 11 & 25 & 7 & 3 & - & 17 & 37 & 1.44 & 1.18 & 1.22 & 2.57 & 6.00 & + & \\
\hline $1 \mathrm{H}-2,106-107$ & 2.56 & Light clay & 10 & 25 & 5 & 3 & - & 21 & 36 & 1.60 & 1.10 & 1.45 & 2.77 & 5.26 & + & + \\
\hline $1 \mathrm{H}-2,120-121$ & 2.70 & Black clay & 10 & 22 & tr. & 15 & 10 & 15 & 28 & 1.45 & 1.00 & 1.45 & 2.07 & 4.64 & + & \\
\hline $1 \mathrm{H}-2,127-128$ & 2.77 & Light clay & 7 & 17 & 12 & 3 & - & 14 & 30 & 1.54 & 1.44 & $\begin{array}{l}1.07 \\
1.07\end{array}$ & 2.55 & $\begin{array}{l}6.56 \\
6.56\end{array}$ & + & \\
\hline $1 \mathrm{H}-2,136-137$ & 2.86 & Black clay & 10 & 28 & 5 & 2 & - & 16 & 39 & 1.21 & 1.00 & 1.21 & 2.09 & 4.33 & + & \\
\hline $1 \mathrm{H}-2,146-147$ & 2.96 & Light clay & 10 & 28 & 5 & tr. & - & 19 & 38 & 1.39 & 1.09 & 1.28 & 2.19 & 5.96 & + & + \\
\hline $1 \mathrm{H}-3,3-4$ & 3.03 & Black clay & 11 & 27 & 5 & tr. & - & 21 & 36 & 1.56 & 1.08 & 1.44 & 2.56 & 6.58 & + & + \\
\hline $1 \mathrm{H}-3,12-13$ & 3.12 & Light clay & 10 & 25 & 5 & 2 & - & 16 & 42 & 1.36 & 1.28 & 1.07 & 2.10 & 5.10 & + & + \\
\hline $1 \mathrm{H}-3,20-21$ & 3.20 & Light clay & 11 & 23 & 8 & 2 & - & 18 & 38 & 1.67 & 1.28 & 1.30 & 2.10 & 5.41 & + & \\
\hline $1 \mathrm{H}-3,27-28$ & 3.27 & Black clay & $\begin{array}{l}11 \\
10\end{array}$ & 25 & $\begin{array}{l}\circ \\
5\end{array}$ & tr. & 二 & $\begin{array}{l}10 \\
17\end{array}$ & 43 & 1.41 & $\begin{array}{l}1.20 \\
1.31\end{array}$ & 1.07 & 2.29 & 5.07 & $\begin{array}{l}+ \\
+\end{array}$ & + \\
\hline $1 \mathrm{H}-3,36-37$ & 3.36 & Light clay & 12 & 27 & 3 & $\begin{array}{l}\text { U. } \\
\text { tr. }\end{array}$ & - & 18 & 40 & $\begin{array}{l}1.41 \\
1.39\end{array}$ & $\begin{array}{l}.031 \\
1.09\end{array}$ & 1.28 & 1.94 & 5.45 & $\begin{array}{l}+ \\
+\end{array}$ & + \\
\hline $1 \mathrm{H}-3,44-45$ & 3.44 & Black clay & 12 & 26 & 3 & 2 & - & 20 & 37 & 1.58 & 1.14 & 1.39 & 2.25 & 5.69 & ++ & + \\
\hline $1 \mathrm{H}-3,56-57$ & 3.56 & Light clay & 10 & 23 & 5 & tr. & - & 18 & 44 & 1.52 & 1.52 & 1.00 & 2.75 & 6.15 & + & \\
\hline $1 \mathrm{H}-3,73-74$ & 3.73 & Light clay & 11 & 21 & 8 & 2 & - & 16 & 42 & 1.53 & 1.51 & 1.01 & 2.23 & 5.29 & + & \\
\hline $1 \mathrm{H}-3,80-81$ & 3.80 & Black clay & 10 & 26 & 3 & 2 & - & 15 & 44 & 1.17 & 1.24 & 0.95 & 2.22 & 4.22 & + & \\
\hline $1 \mathrm{H}-3,97-98$ & 3.97 & Light clay & 10 & 20 & 10 & tr. & - & 14 & 46 & 1.45 & 1.76 & 0.82 & 2.20 & 4.96 & + & \\
\hline $1 \mathrm{H}-3,103-104$ & 4.03 & Black clay & 9 & 26 & 15 & tr. & - & 17 & 33 & 1.35 & 1.01 & 1.33 & 2.50 & 5.83 & + & + \\
\hline $1 \mathrm{H}-3,107-108$ & 4.07 & Light clay & 10 & 22 & 15 & tr. & & 16 & 37 & 1.49 & 1.36 & 1.10 & 2.44 & 6.38 & + & \\
\hline
\end{tabular}

Notes: tr. $=$ trace; $+=$ rare; $++=$ common; smectite crystallinity $=17 \AA$ height $/$ peak width at half height on glycolated diffractograms. 
Table 4. Clay mineral composition (in \%) of Amazon Fan sediments in Hole $931 \mathrm{~B}$.

\begin{tabular}{|c|c|c|c|c|c|c|c|c|c|c|c|c|c|c|c|}
\hline \multirow{2}{*}{$\begin{array}{l}\text { Core, section, } \\
\text { interval }(\mathrm{cm})\end{array}$} & \multirow{2}{*}{$\begin{array}{l}\text { Depth } \\
\text { (mbsf) }\end{array}$} & \multirow{2}{*}{$\begin{array}{c}\text { Lithologic } \\
\text { units }\end{array}$} & \multirow{2}{*}{ Chlorite } & & & & & & Gylcol & lated peak he & ght ratios & Smectite & & & Mineral \\
\hline & & & & Illite & $(14 c-14 s)$ & $(10-14 v)$ & Kaolinite & Smectite & $7 \AA / 10 \AA$ & $17 \AA / 10 \AA$ & $7 \AA / 17 \AA$ & crystallinity & Quartz & Feldspar & zones \\
\hline 155-931B- & & & & & & & & & & & & & & & \\
\hline $1 \mathrm{H}-\mathrm{CC}, 34-36$ & 0.34 & UI & 10 & 24 & 5 & - & 20 & 41 & 1.71 & 1.22 & 1.40 & 3.85 & + & + & \\
\hline $2 \mathrm{H}-1,54-56$ & 1.34 & UIIA & 12 & 31 & 5 & tr. & 16 & 36 & 1.21 & 0.95 & 1.27 & 6.58 & + & + & \\
\hline $2 \mathrm{H}-3,55-57$ & 4.35 & & 12 & 26 & 3 & 2 & 19 & 38 & 1.50 & 1.19 & 1.26 & 6.58 & + & + & M I \\
\hline $3 \mathrm{H}-1,56-58$ & 10.86 & & 12 & 27 & 5 & - & 17 & 39 & 1.44 & 1.14 & 1.26 & 6.15 & + & & \\
\hline $3 \mathrm{H}-3,55-57$ & 13.8 & UIIB & 13 & 25 & 5 & - & 17 & 40 & 1.49 & 1.38 & 1.08 & 6.72 & + & & \\
\hline $4 \mathrm{H}-1,55-57$ & 20.35 & & 14 & 28 & - & tr. & 19 & 39 & 1.64 & 1.10 & 1.49 & 5.85 & + & & \\
\hline $4 \mathrm{H}-3,55-57$ & 23.3 & & 12 & 28 & $\overline{3}$ & 2 & 17 & 38 & 1.35 & 1.10 & 1.23 & 6.00 & + & & \\
\hline $5 \mathrm{H}-1,53-55$ & 29.83 & & 13 & 28 & 5 & - & 19 & 35 & 1.46 & 0.93 & 1.57 & 4.92 & + & & \\
\hline $5 \mathrm{H}-3,57-59$ & 32.87 & & 12 & 27 & 10 & tr. & 18 & 33 & 1.39 & 1.05 & 1.32 & 6.72 & + & + & \\
\hline $6 \mathrm{H}-1,54-56$ & 39.34 & & 13 & 30 & 5 & - & 18 & 34 & 1.42 & 0.91 & 1.56 & 5.83 & + & + & (a) \\
\hline $6 \mathrm{H}-3,54-57$ & 42.34 & & 13 & 24 & 8 & 2 & 18 & 35 & 1.58 & 1.11 & 1.42 & 4.93 & + & + & \\
\hline $7 \mathrm{H}-1,55-58$ & 48.85 & & 12 & 25 & 3 & 2 & 16 & 42 & 1.45 & 1.34 & 1.08 & 5.78 & + & + & \\
\hline $7 \mathrm{H}-3,55-57$ & 51.85 & & 13 & 28 & 2 & 3 & 20 & 34 & 1.52 & 1.00 & $\begin{array}{l}1.00 \\
1.52\end{array}$ & 6.88 & $\begin{array}{lll}+ \\
+\end{array}$ & + & \\
\hline $8 \mathrm{H}-1,55-57$ & 58.3 & & 12 & 29 & 5 & tr. & 20 & 34 & 1.52 & 0.92 & 1.65 & 6.15 & + & + & \\
\hline $8 \mathrm{H}-3,47-49$ & 61.27 & & 12 & 29 & 5 & - & 20 & 34 & 1.40 & 1.11 & 1.26 & 5.93 & + & $T$ & \\
\hline $9 \mathrm{H}-1,54-56$ & 67.84 & & 12 & 28 & 5 & 5 & 15 & 35 & 1.17 & 0.94 & 1.24 & 4.73 & + & + & \\
\hline $9 \mathrm{H}-3,53-55$ & 70.83 & & 11 & 25 & 5 & - & 17 & 42 & 1.43 & 1.29 & 1.11 & 5.17 & & & \\
\hline $10 X-1,54-56$ & 77.34 & & 12 & 25 & 8 & 2 & 21 & 32 & $\begin{array}{l}1.69 \\
\text { (1) }\end{array}$ & 1.01 & 1.67 & 6.30 & + & + & M II \\
\hline $10 \mathrm{X}-3,30-32$ & 80.1 & & 11 & 28 & $\begin{array}{r}\circ \\
10\end{array}$ & 2 & 19 & 32 & 1.51 & 0.90 & 1.68 & 5.76 & + & + & 1011 \\
\hline $11 \mathrm{X}-1,60-62$ & 84.9 & UIIC & 13 & 26 & 5 & tr. & 19 & 37 & 1.58 & 1.04 & 1.52 & 6.74 & + & & \\
\hline $11 \mathrm{X}-3,55-57$ & 87.85 & & 12 & 29 & 3 & 2 & 21 & 33 & 1.52 & 0.97 & 1.57 & 7.12 & + & + & \\
\hline $12 X-1,55-57$ & 94.45 & & 10 & 23 & 8 & 2 & 15 & 42 & 1.33 & 1.37 & 0.97 & 4.34 & + & & \\
\hline $12 X-3,55-57$ & 97.45 & & 14 & 27 & 3 & 2 & 23 & 33 & 1.81 & 1.02 & 1.77 & 6.77 & + & + & \\
\hline $13 \mathrm{X}-1,62-64$ & 104.12 & & 12 & 27 & 3 & 2 & 20 & 36 & 1.64 & 1.05 & 1.56 & 5.36 & + & $T$ & \\
\hline $13 \mathrm{X}-3,83-85$ & 107.33 & & 13 & 28 & 3 & 2 & 20 & 34 & 1.57 & 0.99 & $\begin{array}{l}1.50 \\
1.59\end{array}$ & 6.56 & + & & \\
\hline $14 \mathrm{X}-1,65-67$ & 113.85 & & 11 & 25 & 2 & 3 & 23 & 36 & 1.78 & 1.24 & 1.44 & 7.50 & + & + & \\
\hline $14 \mathrm{X}-3,1-3$ & 116.07 & & 12 & 26 & 8 & 2 & 20 & 32 & 1.54 & 0.99 & 1.56 & 6.40 & + & + & (b) \\
\hline $15 \mathrm{X}-1,66-68$ & 123.46 & & 12 & 26 & 5 & 3 & 19 & 35 & 1.51 & 1.10 & 1.37 & 6.77 & + & + & \\
\hline $15 \mathrm{X}-3,57-59$ & 126.37 & & 13 & 25 & 2 & 3 & 18 & 39 & 1.51 & 1.26 & 1.20 & 6.34 & + & + & \\
\hline $16 \mathrm{X}-3,75-77$ & 136.25 & & 13 & 27 & 10 & tr. & 21 & 29 & 1.64 & 0.89 & 1.84 & 6.40 & + & + & \\
\hline $19 \mathrm{X}-1,57-60$ & 162.07 & UIIC & 13 & 26 & 10 & 2 & 17 & 32 & $\begin{array}{l}1.04 \\
1.48\end{array}$ & $\begin{array}{l}0.09 \\
0.98\end{array}$ & $\begin{array}{l}1.04 \\
1.51\end{array}$ & $\begin{array}{l}0.40 \\
6.00\end{array}$ & $\begin{array}{lll}+ \\
+\end{array}$ & + & \\
\hline $19 \mathrm{X}-3,51-53$ & 165.01 & (lower) & 11 & 27 & 10 & 2 & 18 & 32 & $\begin{array}{l}1.38 \\
1.38\end{array}$ & 0.98 & 1.41 & 7.39 & + & & \\
\hline $21 \mathrm{X}-1,51-53$ & 181.21 & & 12 & 30 & 10 & 2 & 17 & 29 & 1.25 & 0.78 & 1.60 & 6.15 & + & + & \\
\hline $25 X-1,54-56$ & 219.64 & & 12 & 31 & 5 & tr. & 17 & 33 & 1.16 & 0.84 & 1.38 & 5.58 & + & + & M III \\
\hline $25 \mathrm{X}-3,45-47$ & 222.55 & & 10 & 27 & 12 & 3 & 18 & 30 & 1.39 & 0.87 & 1.60 & 5.57 & + & + & \\
\hline $26 \mathrm{X}-1,50-52$ & 229.2 & & 10 & 25 & 15 & - & 20 & 30 & 1.49 & 0.97 & 1.54 & 6.85 & + & + & \\
\hline $27 \mathrm{X}-1,40-42$ & 238.8 & & 12 & 25 & 13 & $\overline{2}$ & 22 & 26 & 1.74 & 0.80 & 2.18 & $\begin{array}{l}0.03 \\
5.60\end{array}$ & $\begin{array}{l}+ \\
+\end{array}$ & $\begin{array}{lll}+ \\
+\end{array}$ & \\
\hline $27 \mathrm{X}-3,46-48$ & 241.86 & & 11 & 30 & 8 & 2 & 18 & 31 & 1.24 & 0.82 & 1.51 & 6.00 & + & & \\
\hline $28 \mathrm{X}-3,52-55$ & 250.49 & & 11 & 32 & 8 & 2 & 22 & 25 & 1.42 & 0.64 & 2.22 & 5.73 & + & & \\
\hline $28 \mathrm{X}-5,50-52$ & 253.47 & & 11 & 26 & 15 & tr. & 18 & 30 & 1.53 & 0.92 & 1.66 & 5.57 & + & & \\
\hline $29 \mathrm{X}-2,61-63$ & 259.81 & & 13 & 28 & 8 & 2 & 18 & 31 & 1.44 & 0.93 & 1.55 & 6.08 & + & + & \\
\hline $29 \mathrm{X}-4,55-57$ & 262.75 & & 12 & 26 & 15 & tr. & 15 & 32 & 1.31 & 0.92 & 1.42 & 5.76 & + & + & \\
\hline $31 \mathrm{X}-1,68-70$ & 277.58 & & 14 & 25 & 5 & 3 & 21 & 32 & 1.73 & 0.98 & 1.77 & 5.27 & $\begin{array}{l}+ \\
+\end{array}$ & + & \\
\hline $31 \mathrm{X}-3,90-92$ & 280.8 & & 12 & 27 & 8 & 2 & 19 & 32 & 1.56 & 0.95 & 1.64 & 6.50 & + & + & \\
\hline $32 \mathrm{X}-1,54-56$ & 287.14 & & 8 & 23 & 10 & tr. & 20 & 39 & 1.56 & 1.36 & 1.15 & 6.38 & + & $T_{T}$ & \\
\hline $32 \mathrm{X}-3,54-56$ & 290.14 & & 12 & 27 & 8 & 2 & 20 & 31 & 1.53 & 0.87 & 1.76 & 6.14 & + & & M IV \\
\hline $33 \mathrm{X}-1,55-68$ & 299.86 & & 11 & 26 & 15 & tr. & 15 & 33 & 1.28 & 1.01 & 1.27 & 6.15 & + & + & \\
\hline $34 \mathrm{X}-1,55-57$ & 306.35 & UIII & 13 & 32 & 5 & $\begin{array}{l}\text { ti. } \\
\text { tr. }\end{array}$ & 21 & 29 & 1.39 & 0.78 & 1.78 & 7.15 & + & + & \\
\hline $34 \mathrm{X}-3,55-57$ & 309.35 & & 11 & 27 & 5 & tr. & 14 & 43 & 1.20 & 1.25 & 0.96 & $\begin{array}{l}1.13 \\
5.69\end{array}$ & + & + & \\
\hline $35 \mathrm{X}-1,40-42$ & 315.9 & & 10 & 34 & 10 & tr. & 19 & 27 & 1.20 & 0.71 & $\begin{array}{l}1.69 \\
1.69\end{array}$ & 7.25 & + & + & \\
\hline $35 \mathrm{X}-2,40-42$ & 317.4 & & 11 & 31 & 5 & $\begin{array}{l}\text { tr. } \\
\text { tr. }\end{array}$ & 18 & 35 & 1.31 & 0.88 & 1.49 & 5.38 & + & + & \\
\hline $35 \mathrm{X}-3,43-45$ & 318.93 & & 10 & 27 & 10 & 15 & 18 & $(*)$ & 1.40 & $(*)$ & $(*)$ & 2.73 & + & + & \\
\hline $36 \mathrm{X}-1,52-54$ & 325.62 & & 11 & 24 & 15 & tr. & 19 & 31 & 1.35 & 0.97 & 1.39 & 4.69 & + & + & \\
\hline $36 \mathrm{X}-3,52-54$ & 328.62 & & 10 & 22 & 12 & 3 & 17 & 36 & 1.61 & 1.26 & 1.28 & 6.24 & + & & \\
\hline $37 \mathrm{X}-1,61-63$ & 335.41 & & 10 & 28 & 8 & 2 & 19 & 33 & 1.37 & 0.90 & $\begin{array}{l}1.20 \\
1.52\end{array}$ & 4.26 & + & & \\
\hline $37 \mathrm{X}-3,61-63$ & 338.41 & & 10 & 24 & 8 & 2 & 25 & 31 & 1.98 & 1.13 & 1.75 & 8.37 & ++ & + & \\
\hline $38 \mathrm{X}-1,55-57$ & 344.95 & UIII & 10 & 36 & 5 & tr. & 21 & 28 & 1.20 & 0.65 & 1.85 & 5.85 & + & + & \\
\hline $38 \mathrm{X}-3,55-57$ & 347.95 & (lower) & 12 & 31 & 10 & tr. & 20 & 27 & 1.39 & 0.6 & 2.04 & 5.23 & + & & \\
\hline $39 \mathrm{X}-1,67-69$ & 354.77 & UVA & 10 & 29 & 10 & tr. & 25 & 25 & 1.61 & 0.64 & 2.52 & 4.62 & + & & \\
\hline $39 \mathrm{X}-3,66-68$ & 357.76 & & 12 & 31 & 10 & tr. & 20 & 27 & 1.41 & 0.69 & 2.04 & 5.67 & + & & \\
\hline $40 \mathrm{X}-3,50-52$ & 366.46 & & 13 & 32 & 3 & 2 & 20 & 30 & $\begin{array}{l}1.41 \\
1.39\end{array}$ & $\begin{array}{l}0.09 \\
0.52\end{array}$ & 2.67 & $\begin{array}{l}.01 \\
6.18\end{array}$ & $\begin{array}{lll}+ \\
+\end{array}$ & + & \\
\hline $40 X-5,56-58$ & 369.52 & & 12 & 30 & 10 & tr. & 22 & 26 & 1.53 & 0.68 & 2.25 & 6.78 & + & + & \\
\hline $41 \mathrm{X}-2,62-64$ & 375.52 & & 10 & 26 & 12 & 3 & 20 & 29 & 1.50 & 0.84 & 1.79 & 5.36 & + & & \\
\hline $41 \mathrm{X}-4,62-64$ & 378.52 & & 12 & 31 & 12 & tr. & 21 & 24 & 1.41 & 0.61 & 2.31 & 6.00 & + & + & M V \\
\hline $42 \mathrm{X}-1,54-56$ & 383.44 & & 13 & 31 & 10 & 3 & 18 & 25 & 1.36 & 0.69 & 1.97 & 6.61 & + & + & \\
\hline $42 X-3,55-57$ & 386.45 & UVB & 12 & 29 & 12 & tr. & 16 & 31 & 1.26 & 0.79 & 1.59 & 5.30 & + & & \\
\hline $43 \mathrm{X}-2,62-65$ & $\begin{array}{l}500.43 \\
394.09\end{array}$ & UVD & 10 & 28 & 13 & 2 & 20 & 27 & 1.38 & 0.77 & 1.79 & 5.84 & $\begin{array}{lll}+ \\
+\end{array}$ & & \\
\hline $43 \mathrm{X}-4,44-46$ & 396.91 & & 13 & 27 & 12 & 3 & 17 & 28 & 1.39 & 0.77 & 1.81 & 4.88 & + & + & \\
\hline $44 \mathrm{X}-2,55-57$ & 402.92 & & 13 & 30 & 10 & 2 & 20 & 25 & 1.41 & 0.62 & 2.27 & 4.61 & + & + & \\
\hline $44 X-4,55-57$ & 405.86 & & 13 & 32 & 12 & tr. & 21 & 22 & 1.41 & 0.56 & 2.52 & 6.09 & + & & \\
\hline $45 \mathrm{X}-1,44-46$ & 412.04 & & 13 & 32 & 12 & tr. & 18 & 25 & 1.22 & 0.66 & 1.85 & 7.33 & + & + & \\
\hline $45 \mathrm{X}-3,47-49$ & 415.07 & & 13 & 30 & 15 & tr. & 18 & 24 & 1.41 & 0.63 & 2.24 & 5.68 & + & + & \\
\hline
\end{tabular}

Note: $-=$ no data; tr. = trace $+=$ rare $++=$ common; smectite crystallinity $=17$ A peak height $/$ peak width at half height on glycolated diffractograms; $(*)=20 \%$ illite-smectite $14 \mathrm{v}-$ $14 \mathrm{~s}$.

rite-smectite mixed layers mainly derive from an incomplete weathering of chlorite, and (3) the feldspars included in the $<2-\mu \mathrm{m}$ fraction of the fan sediments are much less abundant than in the shallower shelf deposits (Milliman et al., 1975).

The bibliographic data indicate that the river sediments of the Amazon Basin System are often dominated by illite associated with chlorite in most of the upstream Andean tributaries (e.g., Bolivian Amazon; Guyot, 1992), even if the clay mineral assemblage may be locally more diversified (Gibbs, 1967). In these mountainous areas where the physical weathering and the erosion of rock-derived products predominate, kaolinite issuing from chemical weathering frequently represents a detrital component of secondary abundance, as 


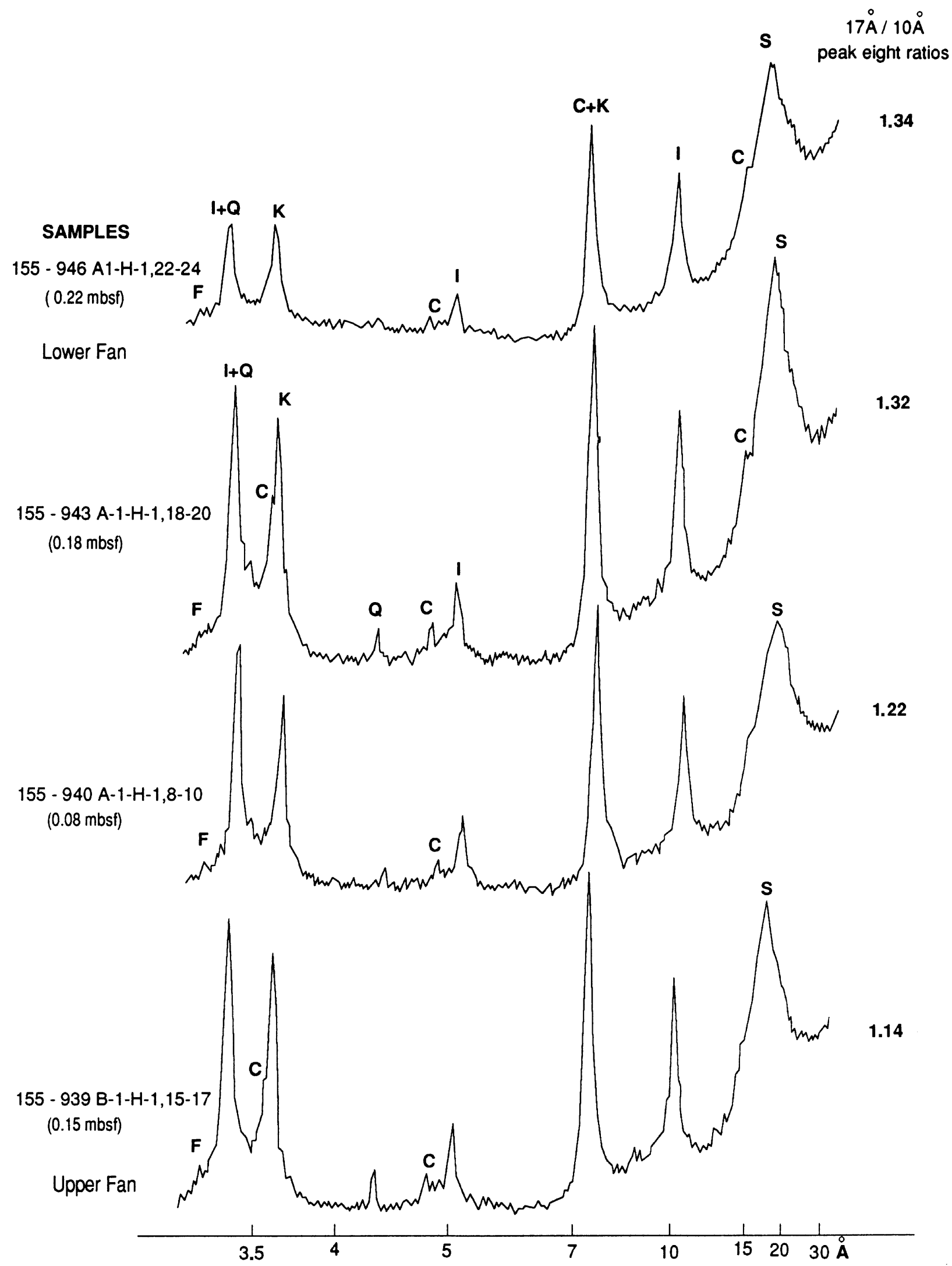

Figure 3. X-ray diffraction traces (glycolated samples) of the $<2-\mu \mathrm{m}$ fraction of recent sediments from ODP Leg 155 drilling sites and related $17 / 10 \AA$ ratio values. $\mathrm{I}=$ illite; $\mathrm{C}=$ chlorite; $\mathrm{K}=$ kaolinite; $\mathrm{S}=$ smectite; $\mathrm{Q}=$ quartz; $\mathrm{F}=$ feldspar. 
well as smectite, which is only locally present. When moving downstream along the river basin, kaolinite and smectite amounts increase (Gibbs, 1967; Johnsson and Meade, 1990), even if the kaolinite flux locally decreases as the result of preferential settling (Irion, 1984).

\section{Spatial Distribution}

The surficial sediments deposited along the present-day channel of the middle to lower Amazon Fan display very few changes in the clay-mineral distribution, over a distance of about $260 \mathrm{~km}$ (Table 2; Fig. 3). The only suspected change consists in a slight proximal-todistal increase of the smectite to illite relative abundance, recorded from Site 939 to Site 946 (i.e., $17 \AA$ Å/10 Å peak height ratio). Even this trend, however, remains partly questionable, suggesting that most of the clay mineral sorting processes develop on a greater distance than a few hundred kilometers (see Gibbs, 1977) and/or that they occur preferentially in the upper fan rather than in the middle to lower fan. The latter hypothesis is supported by the fact that most winnowing processes in the modern submarine Amazon sedimentary system occur in the middle shelf domain (Gibbs, 1967; Barreto et al., 1975; Milliman et al. 1975).

The clay mineral distribution in surficial sediments sampled along an east-west transect normal to the Amazon Channel (Fig. 4) is characterized by a significant and continuous increase of smectite relative abundance (35\%-42\% of the clay fraction from Sites 931 to 942 ; Fig. 1; Table 2). This east-to-west trend corresponds to ODP sites drilled in the Amazon Channels called 5 and Purple (Manley and Flood, 1988), which operated successively in the course of the time. Such a clay mineral trend points to the existence of active winnowing processes in a direction within the oceanic domain that is normal to that of the river flux. It is assumed to result from sorting mechanisms determined by the North Brazil Current, which is annually responsible for the westward transportation of about $10^{8}$ metric tons of suspended matter (Allersma, 1971; Nittrouer et al., 1986, 1995).

\section{Late Glacial Distribution}

The clay mineral associations identified in the very surficial sediments (i e., a few decimeters to less than $1 \mathrm{~m}$ depth) often differ notably from those characterizing the deeper sediments. This is especially the case at Holes 930B, 936A, 937B, 940B, 945A (Table 2) where uppermost samples are enriched in smectite and/or kaolinite and the subsurface samples contain more illite and chlorite. This trend is nevertheless not systematic, some sites showing less smectite in uppermost than in subsurface sediments (e.g., Holes 934A, 938A, 944A); but in these cases, the uppermost levels contain more mixedlayer clays (chlorite-smectite), that is, expandable structures belonging to the same mineralogical group as smectite. As a consequence, weathering-derived clay minerals occur more abundantly in the surficial calcareous oozes than in the underlying silty clays. This uphole enrichment of smectite and/or kaolinite and of random mixed layers was probably determined by increased weathering processes on land (Chamley, 1989) or by the development of arid periods diminishing the continental erosion as suggested by Pujos et al. (in press).

\section{HIGH-RESOLUTION DISTRIBUTION IN HOLOCENE CYCLIC DEPOSITS, HOLE 934A}

\section{Lithology and Stratigraphy}

Hole 934A is located in the central part of the Amazon Fan in a cutoff meander bend of the Amazon Channel (Fig. 1; Shipboard Scientific Party, 1995b). It penetrated coarse channel floor sediments at $108.8 \mathrm{mbsf}$ that are overlain by sediments accumulated after the meander loop was abandoned. In particular, the uppermost part of the hole penetrated numerous superimposed muddy turbidite overspills, indicating the turbidite-related processes continued at the beginning of the last interglacial stage. This preserved interval was an opportunity to investigate, in detail, the clay mineral pattern distribution and significance in a thin time series that is well constrained biostratigraphically. Forty plugs were sampled within the four surficial meters, representing the muddy turbidite packet and the overlying hemipelagic carbonates, both reported to Holocene time. The sample spacing is 7-20 cm in Section 1 and 3-17 cm in Sections 2 and 3 (Fig. 5). Based on facies characteristics, shipboard sedimentological investigations distinguished between two lithologic units (Fig. 5), Unit I and Unit II.

Unit I (0-0.87 mbsf) corresponds to the carbonate-rich interval that drapes the entire fan. From top to base, three distinctive facies have been determined: brown foraminifer-nannofossil ooze $(0-0.47$ mbsf) partly cemented by an iron-rich crust in the lowermost part; dark gray nannofossil silty clay $(0.47-0.63 \mathrm{mbsf})$ with abundant pteropods at the base; and greenish gray foraminifer-nannofossil clay (0.63-0.87 mbsf) bounded at the base by a $1-\mathrm{cm}$-thick iron-rich crust. The last two intervals characterize the suspension settling of diluted hemipelagic mud suddenly interrupted by a muddy turbiditic event carrying pteropod shells below the aragonite compensation depth. A detailed microfossil study based on the identification and abundance of nannofossils and foraminifers (Shipboard Scientific Party, 1995b) suggests that Unit I was deposited in the last $3.7 \mathrm{k} . \mathrm{y}$.

Unit II, Subunit IIA (0.87-7.70 mbsf) consists of dark olive-gray clay and silty clay with black color banding at a scale of $1 \mathrm{~mm}$ to several centimeters. Only the upper $3.30 \mathrm{~m}$ of this unit, corresponding to the Holocene transitional facies, have been investigated. The top of this interval consists of intensely bioturbated and mottled dark gray clay (0.87-1.41 mbsf) containing a 2-cm-thick indurated green crust at its base. This facies overlies color-banded silty clay forming 10 to 25-cm-thick cycles. Each cycle forms a fining-upward, sharp-based sequence. The lower part is mainly composed of black, hydrotroilite stained and often thinly laminated silt-rich silty clay. It is overlain by a dark gray slightly mottled and burrowed nannofossil-bearing silty clay. Carbonate content ranges from $1.6 \%$ to $3.5 \%$. This interval is interpreted as stacked, thin, fine-grained turbidite sequences. The fine-grained turbidites appear to coincide with the sea-level rise observed between 11 and $\sim 8 \mathrm{ka}$ on the Site 942 isotope curve proposed by Showers et al. (this volume).

\section{Clay Mineralogy}

\section{General Data}

The Holocene clay mineral assemblage comprises dominant smectite $(30 \%-50 \%)$, illite $(15 \%-30 \%)$, kaolinite $(15 \%-25 \%)$, chlorite $(8 \%-13 \%)$, and random mixed layers (trace amounts to $20 \%$ ). The illite-vermiculite mixed layers $(10-14 \mathrm{v})$, which are generally very rare relative to chlorite-smectite $(14 c-14 s)$, occur locally as significant components of the clay fraction (Sample 155-934A-1H-1, 26-28 cm, 74-75 cm, and 132-134 cm; Sample 155-934A-1H-2, $120-121 \mathrm{~cm})$, and are then associated with chlorite-vermiculite types $(14 c-14 v)$. Section 1 displays two clay mineral zones that correspond to the upper part of lithologic Subunit IIA and to lithologic Unit I (Table 3; Fig. 5).

Clay mineral zone M I (4.07-1.52 mbsf; Sample 155-934A-1H3, 107-108 cm, to Sample 155-934A-1H-2, 2-3 cm; 11-9 k.y.). This zone correlates with the alternating light and dark cycles of lithologic Subunit IIA (silty clay), but the clay mineral variations are totally independent of the color alternation. The relative abundance of illite and chlorite, two rock-derived minerals, is larger than expected for the Amazon Fan (e.g., $17 \AA / 10 \AA$ ratio), whereas that of kaolinite is lower (e.g., $3.57 \AA / 3.54 \AA$ ratio), and the vermiculite-bearing mixed layers are very rare (except at Sample 155-934A-1H-2, 120-121 cm). The lowermost part of this mineralogical zone comprises noticeable amounts of chlorite-smectite mixed layers and minimum amounts of 


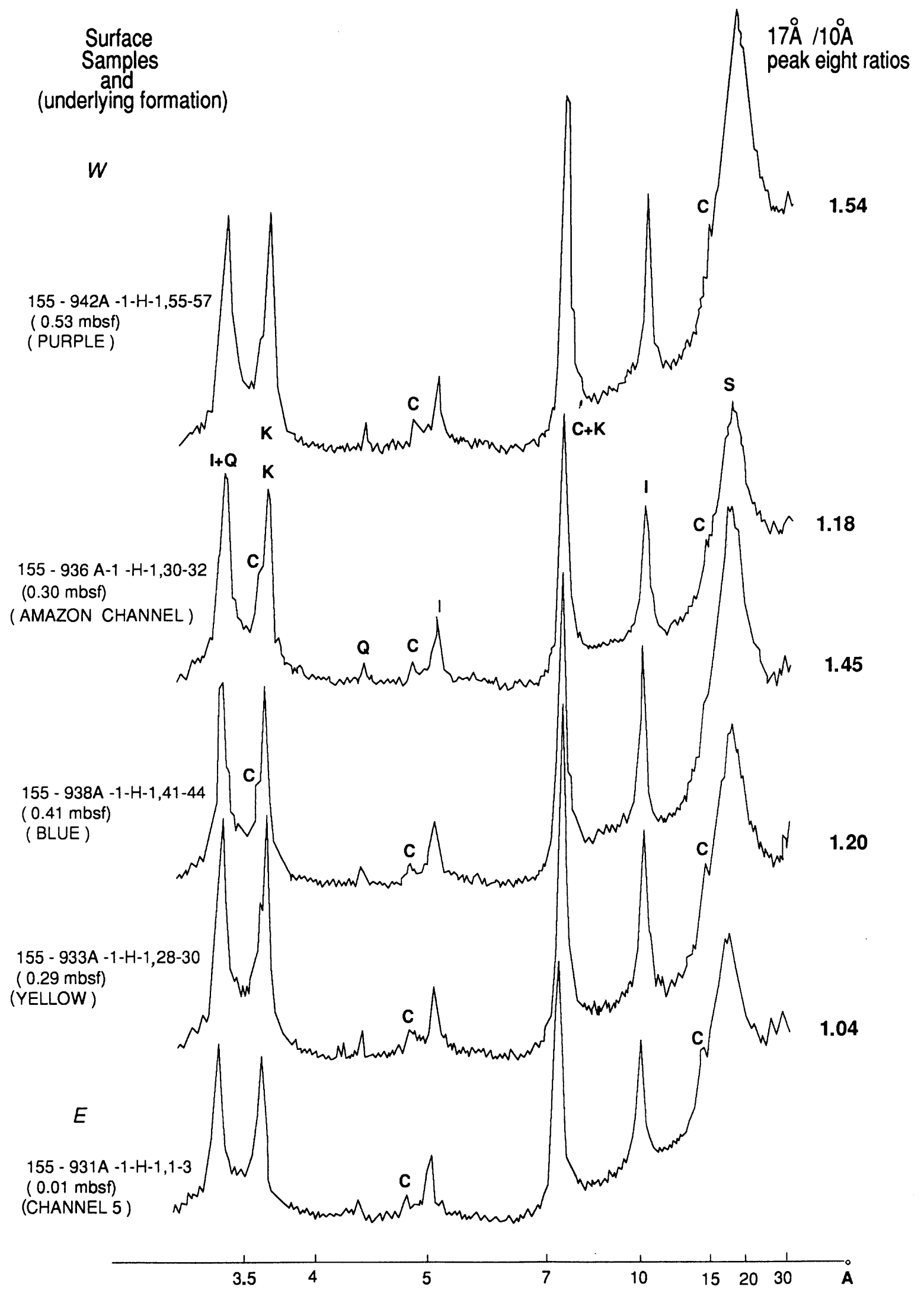

Figure 4. X-ray diffraction traces (glycolated samples) of the $<2-\mu \mathrm{m}$ fraction from surficial Amazon Fan sediments on an east-west transect and related $17 \AA / 10$ $\AA$ ratio values. $\mathrm{I}=$ illite; $\mathrm{C}=$ chlorite; $\mathrm{K}=$ kaolinite; $\mathrm{S}=$ smectite; $\mathrm{Q}=$ quartz. 


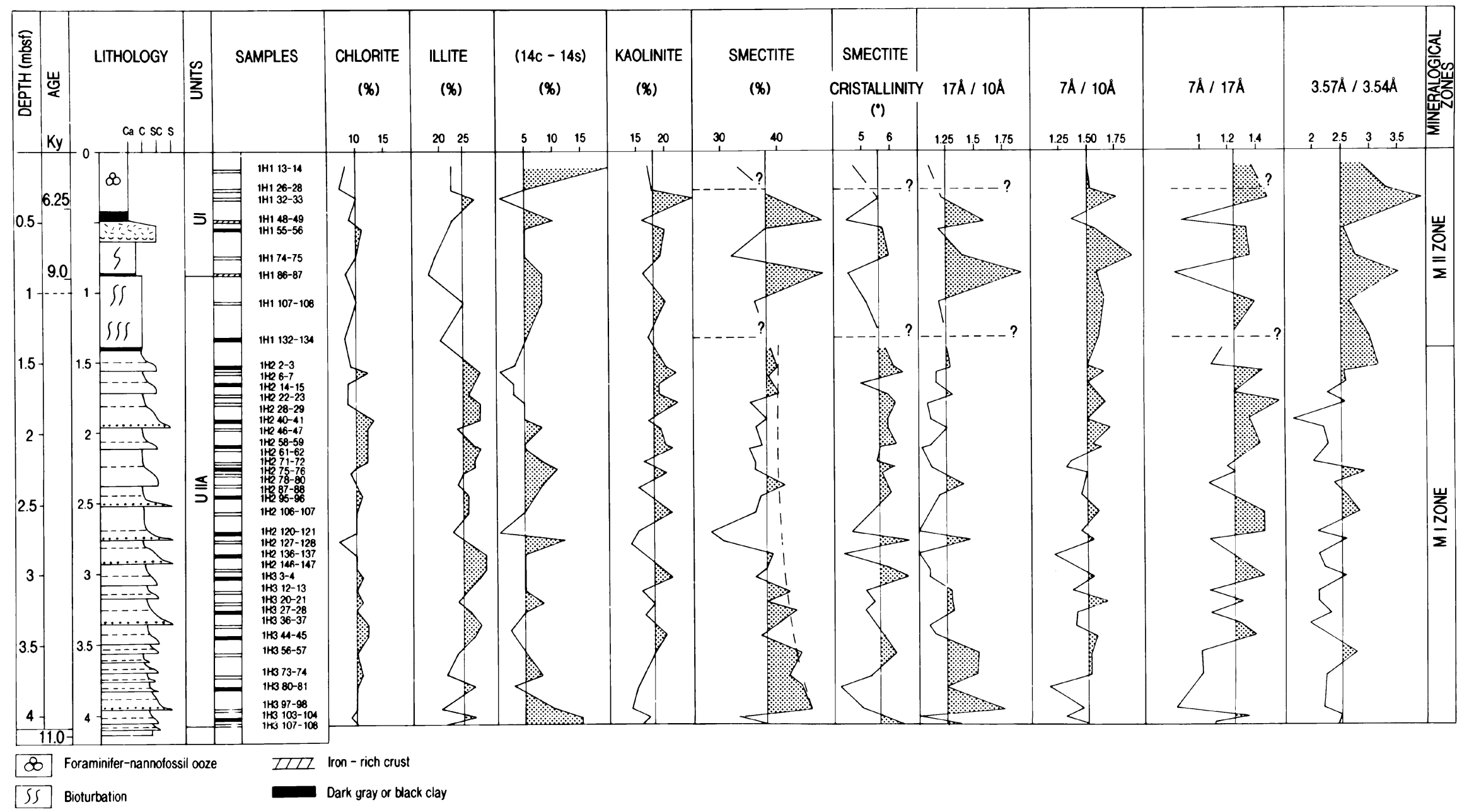

Figure 5. Clay mineral percentages and indices for the sediments at Hole 934A. 
smectite (Samples 155-934A-1H-3, 103-104 cm, and 107-108 cm) except at the base. The abundance of smectite increases just above the base and diminishes progressively upward until the level at Sample 155-934A-1H-2, 120-121 cm, where it becomes more constant.

Clay mineral zone M II (1.52-0.13 mbsf; Samples 155-934A-1H$1,132-134 \mathrm{~cm}$, and $13-14 \mathrm{~cm}$; less than $9 \mathrm{k.y}$.). This zone corresponds to the uppermost part of the lithologic Subunit IIA (bioturbated dark gray clay) and to the entire Unit I (carbonate-bearing clay to clay-bearing calcareous biogenic ooze). The abundance of illite and chlorite is significantly lower than in M I, whereas smectite, kaolinite and random mixed layers are more abundant and display amounts average for the usual values for the area investigated. The vermiculitebearing mixed layers are especially abundant in Samples 155-934A$1 \mathrm{H}-1,132-134 \mathrm{~cm}$ and $26-28 \mathrm{~cm}$, where smectite is almost absent. The levels enriched in metal oxides contain poorly crystalline but abundant smectite.

These results confirm the dominant contribution to the sedimentation of terrigenous material eroded from soils cropping out in the lower part of the Amazon River Basin (smectite, kaolinite). The increased relative abundance of illite and chlorite in uppermost Pleistocene/lowermost Holocene deposits (Subunit IIA, mineralogical zone M I) is probably a specific and temporary supply from upstream mountainous zones of the Andean range, likely determined by active erosion at the end of the last glacial stage. In a similar way, the local increase of incompletely weathered illite and especially chlorite, marked by fairly abundant $(10-14 \mathrm{v}),(14 \mathrm{v}-14 \mathrm{~s})$, and/or $(14 \mathrm{c}-14 \mathrm{~s})$ mixed layers, is probably determined by a temporary supply of detrital material issued from little evolved soils. The particularly high abundance in the oxide-bearing horizons of smectite, a soil-derived clay mineral the deposition of which is favored under very quiet hydrodynamic conditions (Gibbs, 1977; Chamley, 1989; Weaver, 1989), suggests that the lower sedimentation rate responsible for these ferric accumulations results from a reduced terrigenous input due to the sea-level rise. As the alternating light and dark color cycles are independent of the clay mineral distribution, they result from cyclic changes in the biogenic rather than in the terrigenous supply. Finally, one should notice that the fine-grain turbidite inputs occurring during the deposition of lithologic Subunit IIA do not determine any specific modification in the clay mineral supply; this suggests that sorting had already worked during the initial deposition at the river mouth and that resedimentation probably issued from zones marked by hydrodynamic constraints comparable to those of Site 934 .

\section{CLAY MINERAL RECORD OF A QUATERNARY CHANNEL-LEVEE SYSTEM}

\section{Location, Stratigraphy, and Sedimentology}

Site 931 was the most easterly site drilled during Leg 155. It is located on a flat terrace on the western levee of the Channel-levee System 5 (acoustic stratigraphic nomenclature defined by Damuth et al., 1983, and Manley and Flood, 1988), at about $1 \mathrm{~km}$ east of the channel (Fig. 1). Hole 931B was drilled near the levee crest to a maximum depth of $421.3 \mathrm{mbsf}$. It penetrated the complete Channel-levee System 5 at the top of an old channel system capped by a hemipelagic interval and separated from Channel 5 by a 100-m-thick mass-transported deposit (MTD; Fig. 6).

The main objectives of the study were to (1) determine the clay mineral distribution throughout a complete channel-levee system, (2) characterize the clay mineral assemblage of the mass-transported unit, and (3) compare the clay mineral assemblages of Channel 5 to the previous old channel system. About two samples per core were taken along Hole 931B on appropriate lithologies, the sample spacing being 3-6 m.

Five lithologic units were recognized on Hole 931B by the shipboard party (Shipboard Scientific Party, 1995c). Based on complementary shore-based formation microscanner tool string (FMS) interpretations and new biostratigraphic data (Maslin and Mikkelsen, this volume), depositional history and processes have been better constrained and the previous shipboard lithologic units are now replaced in their depositional systems (Fig. 6).

\section{Holocene Interglacial Deposits}

Unit I ( 0 to $0.57 \mathrm{mbsf}$ ) is made up of moderately burrowed brown calcareous clay containing foraminifer and nannofossil ooze. The calcium carbonate content increases uphole from $14 \%$ to $38 \%$. A 3$\mathrm{cm}$-thick diagenetic iron-rich crust occurs at $0.53 \mathrm{mbsf}$ and is recognized in the whole Amazon Fan. These sediments are interpreted as hemipelagic deposits during the last sea-level rise when the turbiditic activity on the Amazon Fan completely vanished.

\section{Transitional Deposits and Uppermost Pleistocene, Post Channel 5, Distal Turbidite Deposits}

The transitional deposits (Subunit IIA, 0.57-6.70 mbsf) consist of slightly to moderately bioturbated and dark olive gray mottled and color-banded clay. The calcium carbonate content drops significantly from $11 \%$ in the upper $10 \mathrm{~cm}$ to $1.5 \%$ in the rest of the subunit, which is interpreted as resulting from the reduction of turbidite input with the sea-level rise. Shipboard studies suggest that the 11 k.y. Z/Y Erickson Zone boundary lies in Subunit IIA (Shipboard Scientific Party 1995c). Post-Channel 5 distal turbidites deposits (Subunit IIB, 6.70-27.50 mbsf) are made up of dark olive-gray silty clay with subtle light and dark color banding. The color banding corresponds to a millimeter- to centimeter-thick alternation of silty clay-clayey silt. The bioturbation expressed by sediment mottling decreases downward throughout the unit. These fine-grained turbidites are attributed to flows originating from successive adjacent channel-levee systems developed after the abandonment of Channel 5 .

\section{Late Pleistocene Channel 5 System}

Piper et al. (Chapter 39, this volume) propose from isotopic, biostratigraphic, and paleomagnetic data that the Channel-levee System 5 developed during glacial Stage 3 (Fig. 6). The Channel-levee System 5 comprises in the upper part levee deposits and in the lower part, high-amplitude reflection packet (HARP) deposits. Channel 5 levee deposits correspond to the first $102.5 \mathrm{~m}$ of Subunit IIC (27.5-199.8 mbsf), consisting of mud with thin to thick beds of silt and sand, the frequency and thickness of which decrease uphole and constitute an overall thinning and fining-upward sequence. The silty clay facies contains color bands and mottling that increase in intensity and frequency with depth. Some silt and sand are graded and/or exhibit parallel or cross-lamination forming Tbde or Tcde turbidites (Bouma, 1962). This sequence is related to the autocyclic buildup of the Channel-levee System 5 by turbidite overspills, which increasingly restricted the coarse fraction deposition within the channel and led to the thinning and fining upward trend. Channel 5 HARP deposits correspond to the last $69.80 \mathrm{~m}$ of Subunit IIC and to the first $38.20 \mathrm{~m}$ of Unit III (199.80-349.25 mbsf). These deposits were poorly recovered during drilling due to the abundance of unconsolidated thick coarse beds. The sediments recovered comprise sand beds with mudclast intervals and are separated by muddier units. On seismic reflection profiles this facies forms characteristic flat, high-amplitude, lobe-like packets (HARPs) that have been recognized at the base of all the channel-levee systems in the middle fan and extend partly to the lower fan (Flood et al., 1991). HARPs are thought to be formed after avulsion of a previously active channel-levee system by rapid aggradation of sheet-flow sand.

\section{Late Pleistocene Debris-Flow Deposits}

These deposits correspond to the lower $111.28 \mathrm{~m}$ of Unit III and are mainly composed of various types of overconsolidated and highly contorted very dark gray clay and silty clay. The deposits comprise 


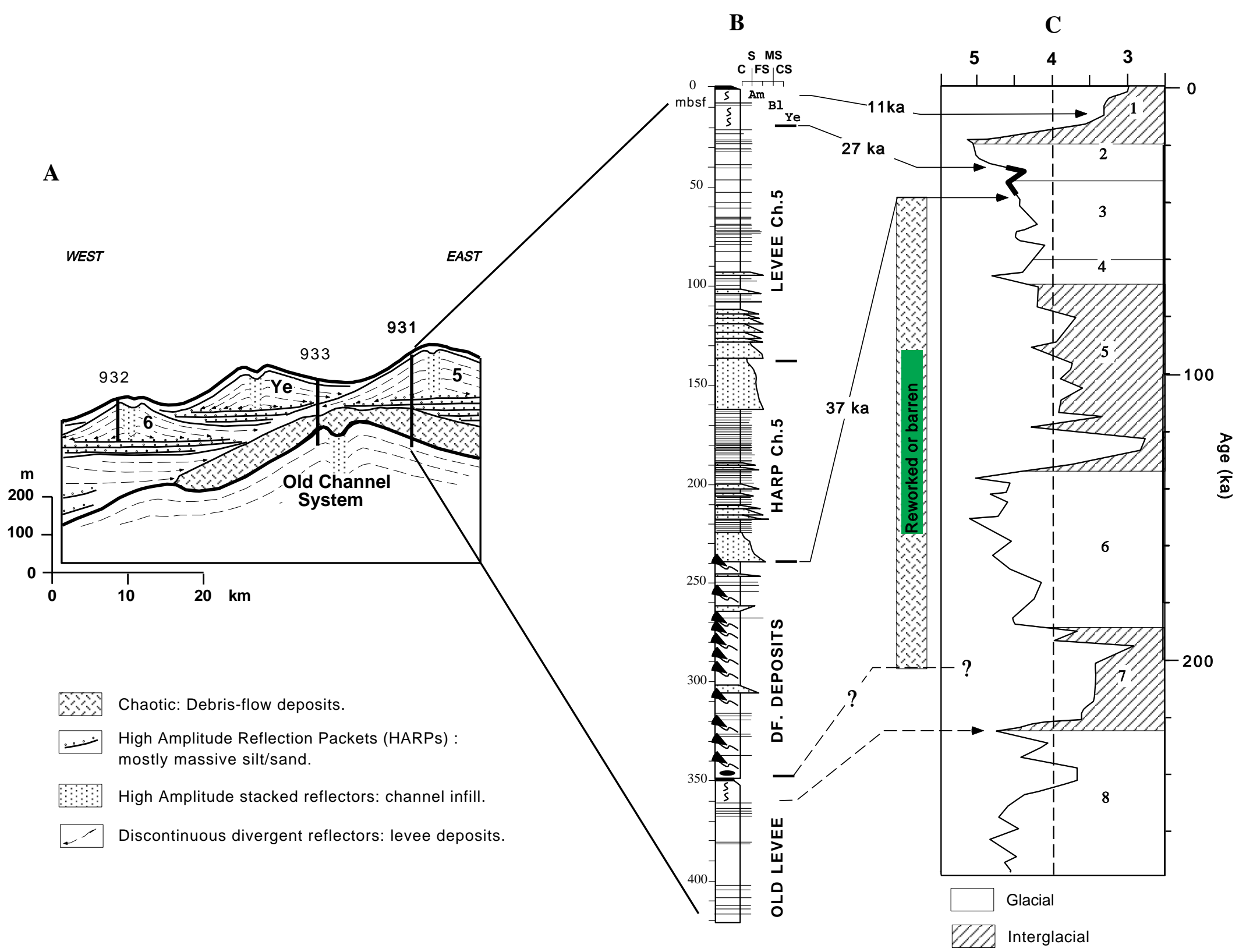

Figure 6. Lithology and stratigraphy for Hole 931B. A. Superimposed channel-levee systems recovered at Hole 931B. $5=$ Channel 5 System; $6=$ Channel 6 System; Ye = Yellow System. B Lithology of Hole 931B. C = clay; $\mathrm{S}=$ silt; FS = fine sand; MS = medium sand; CS = coarse sand. C. Position of the sediments from Hole 931B on the benthic oxygen isotope curve recorded from Site 659, by Maslin and Mikkelsen (this volume). 
disrupted and sheared silt or sand laminae, as well as thin beds forming blebs and lenses of various size, shape and orientation. In places, sandy clay intervals occur with wood and shell fragments. Some packets consist of large clasts, some of them being foraminifer- and nannofossil-rich clay. The lowermost clasts in these debris-flow deposits contain warm-water microfossil assemblages, similar to those of the underlying Unit IV. Debris-flow deposits are well defined on seismic profiles where they form a zone of acoustically incoherent and transparent facies at the base of the Upper Levee Complex. They are considered as mass-transported slides and debris-flows, originating in part from the upper slope. Biostratigraphic data (Maslin and Mikkelsen, this volume) indicate that the reworked material of the middle part of the debris-flow deposits is younger than 85 k.y. and older than 40 k.y.

\section{Old Interglacial Deposits}

These middle Pleistocene deposits of Unit IV (349.25-349.46 mbsf), form a thin horizon of intensively bioturbated, dark gray foraminifer-nannofossil-bearing clay with less than 35\% calcium carbonate. The warm-water foraminifers identified are reported to the oxygen isotope Stage 7 (Maslin and Mikkelsen, this volume). This horizon is attributed to a hemipelagic settling during the interglacial highstand of sea level.

\section{Top of the Bottom Levee Complex}

Middle Pleistocene, Unit V (349.46-421.30 mbsf), can be divided into old transitional deposits (Subunit VA, 349.46-360.87 mbsf), made up of faintly mottled and color banded, very dark gray silty clay containing scattered foraminifers reported to isotope Stage 7 (Maslin and Mikkelsen, this volume). This facies is attributed to diluted terrigenous input capping the abandoned old channel system during the sea-level rise contemporaneous to isotope Stage 7. The levee deposits of the Bottom Levee Complex (Subunit VB, 360.87 mbsf; bottom of hole at $421.30 \mathrm{mbsf}$ ) consist of moderately bioturbated dark gray silty clay with silt laminae and thin beds. Some of the beds are graded or cross-bedded, indicating decelerated currents and tractive processes. This facies assemblage is attributed to the gradual construction of the Bottom Levee Complex by overspill turbidites, the lack of nannofossils in this deepest interval precludes to determine a precise biostratigraphic age.

\section{Clay Mineralogy \\ Clay Mineral Distribution and Zonation}

The clay mineral study of Hole 931B is based on X-ray diffraction analyses of 73 clay-rich sediments sampled between 0.34 mbsf (Holocene) and $415.07 \mathrm{mbsf}$ (middle Pleistocene). All shipboard lithologic units have been sampled except Unit IV, which is only $0.21 \mathrm{~cm}$ thick (Fig. 7; Table 4).

The clay mineral assemblages are dominated by either illite $(20 \%-35 \%)$ or smectite $(20 \%-45 \%)$, the latter species being on average less abundant than in the surficial Holocene sediments of the Amazon Fan. The associated clay minerals include kaolinite (15\%$25 \%)$, chlorite $(8 \%-15 \%)$, and random mixed layers that are either expandable $(14 \mathrm{c}-14 \mathrm{~s}, 5 \%-15 \%)$ or not $(10-14 \mathrm{v}$, trace amounts). Moderate but significant variations occur along the sedimentary column (Fig. 7). Illite is abundant at the base of the series up to 345 mbsf. Random mixed layers (14c-14s) are especially well represented in the lower $250 \mathrm{~m}$, and smectite becomes dominant from $345 \mathrm{~m}$ to the top of the hole. We therefore propose five successive mineralogical zones from the base to the top of the Quaternary series.

Clay mineral Zone V (415-345 mbsf, Samples 155-931B-45X-3, 47-49 cm, to 155-931B-38X-1, 55-57 cm; middle Pleistocene). This zone corresponds to lithologic Subunits V and IV (Bottom Levee Complex) and includes the base of Unit III (debris-flow deposits). Il- lite (30\%) predominates on smectite (25\%), the $17 \AA / 10 \AA$ ratio being lower than 1 (Fig. 7; Table 4). Chlorite presents sharp X-ray diffraction peaks indicative of a well-crystalline state $(14 \mathrm{c}-14 \mathrm{~s})$. Random mixed layers are fairly abundant $(10 \%-15 \%)$ and regularly distributed. The relative abundance of kaolinite tends to increase uphole in very slight proportions ( $7 \AA / 17 \AA$ ratio often higher than 2 ). The base of the debris-flow deposits investigated on a single sample (155931B-38X-3, 55-57 cm), displays a clay mineral composition identical to the top of lithologic Unit V, suggesting that the first resedimented materials originated from the reworking of the Bottom Levee Complex.

Clay mineral Zone IV (339-239 mbsf, Samples 155-931B-37X-3, 61-63, cm to 155-931B-27X-1, 40-42 cm; late Pleistocene). This clay mineral zone roughly corresponds to most of the debris-flow deposits and base of the HARP Channel 5 System. Smectite predominates on illite, the relative abundance of the latter species decreasing relative to the former together with that of kaolinite and chlorite. Random mixed layers are present in variable amounts but at average higher percentage values than in Zone V. The amounts of the different clay mineral groups fluctuate significantly in this zone, which could result from the development of reworking processes possibly implying different source formations. For instance, the smectite abundance and crystalline state successively increase and decrease and the mineral is locally replaced by vermiculitic random mixed layers $(10-14 \mathrm{v}$ and $14 \mathrm{v}-14 \mathrm{~s} ; 20 \%)$.

Clay mineral Zone III (229-136 mbsf, Samples 155-931B-26X-1, 50-52 cm, to $155-931 \mathrm{~B}-16 \mathrm{X}-3,75-77 \mathrm{~cm}$; late Pleistocene). This zone corresponds to the lower part of the lithologic Subunit IIC and to the uppermost part of Unit III (HARP Channel-levee System 5) that is dominated by sandy lithofacies and has been little sampled (Fig. 7). The results are therefore only partly reliable. They show clay associations fairly similar to those of Zone IV, with comparable amounts of smectite and illite, and significant amounts of random mixed layers $(14 \mathrm{c}-14 \mathrm{~s} ; 5 \%-15 \%)$.

Clay mineral Zone II (127-30 mbsf, Samples 155-931B-15X-3, $57-59 \mathrm{~cm}$, to $155-931 \mathrm{~B}-5 \mathrm{H}-1,53-55 \mathrm{~cm}$; late Pleistocene). The upper part of the Subunit IIC (Channel 5 levee formation) correlates with this zone and displays clay mineral assemblages distinct from those of Subunit III. Smectite is systematically dominant relative to illite (see $17 \AA / 10 \AA$ ratio), and especially mixed-layer amounts decrease. The lower part of this zone (Samples 155-931B-15X-3, 57-59 $\mathrm{cm}$ to $155-931 \mathrm{~B}-10 \mathrm{X}-1,54-56 \mathrm{~cm}$ ) contains slightly more abundant kaolinite (e.g., $7 \AA / 10 \AA$ and $7 \AA / 17 \AA$ ratios), whereas the upper part of the zone is depleted in this mineral.

Clay mineral Zone I (23-0.3 mbsf, Samples 155-931B-4H-3, 55$57 \mathrm{~cm}$, to $155-931 \mathrm{~B}-1 \mathrm{H}-\mathrm{CC}, 34-36 \mathrm{~cm}$; uppermost Pleistocene to Holocene). Sparsely sampled, this zone contains in the clay fraction abundant smectite (more than 35\%), which resembles the average contents of this mineral in surface sediments of the Amazon Fan. Smectite becomes less crystalline in the more surficial samples (Unit I), where kaolinite is slightly more abundant than in Subunit IIA.

\section{Discussion}

The temporal variations of the clay mineral assemblages at Hole 931B display various paleoenvironmental modifications having occurred in the Amazon River Basin System and successively registered in the Quaternary sediments. The opposite behavior of smectite and illite, that is, the two dominant species, expresses the control of erosion processes on downstream soils and rocky substrates, respectively. The variations of kaolinite, another dominantly soil-derived species, are parallel to those of either smectite or illite and therefore provide useful information on the respective influence of climate or tectonics (Fig. 2; Chamley, 1989). It is possible to propose the following hypotheses about the paleoenvironmental evolution of the continental domain subject to Quaternary weathering and erosion processes. 


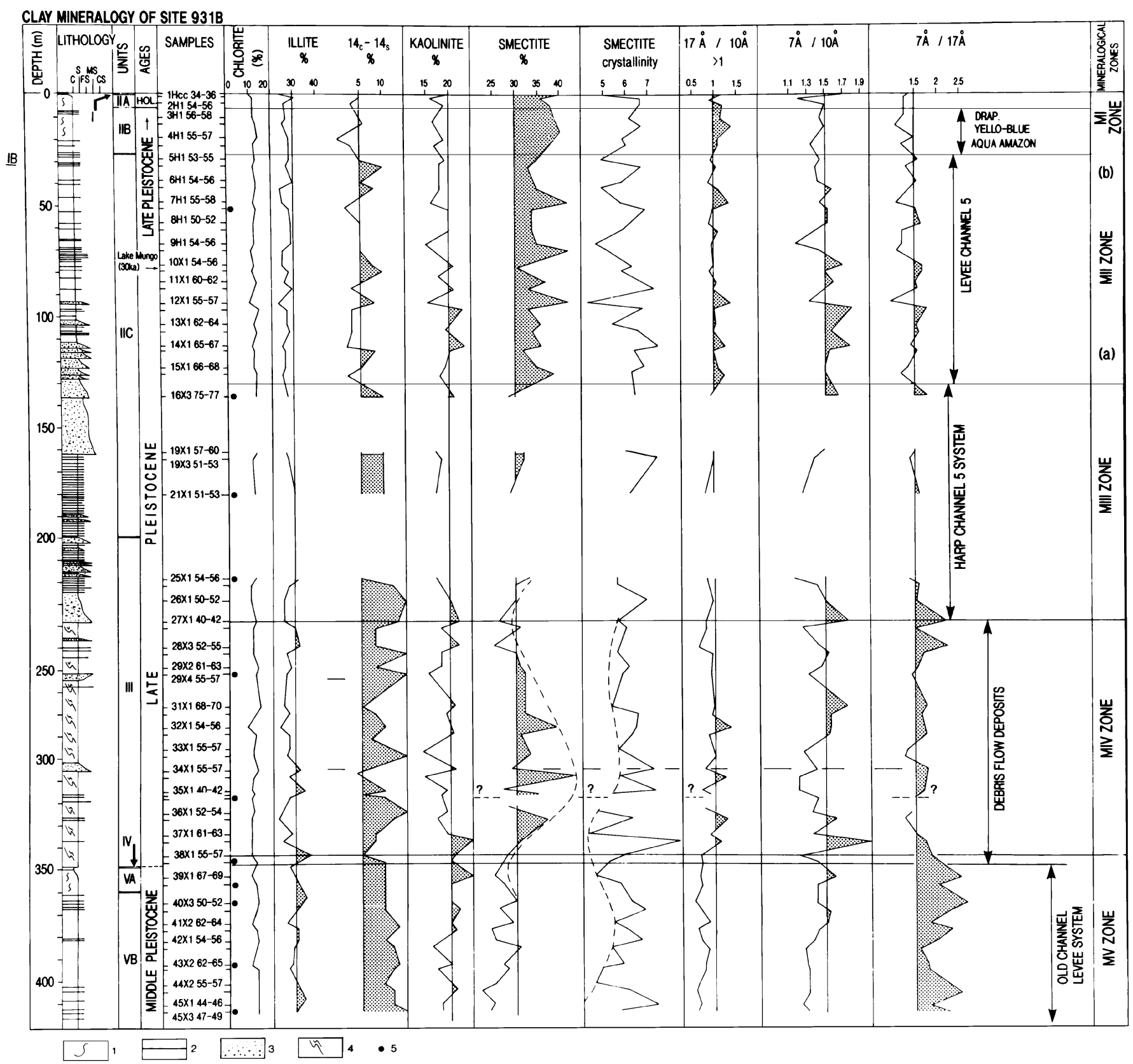

Figure 7. Clay mineral percentages and indices in the $<2-\mu \mathrm{m}$ fraction of sediments at Hole 931B. $1=$ bioturbated; $2=$ laminated; $3=$ graded bed; $4=$ deformed bed; $5=$ grain size analysis.

\section{Clay Mineral Zone V, Middle Pleistocene}

The lower part of the series investigated displays the highest amounts of illite and chlorite, two minerals being dominantly inherited from upstream geological substrates (i.e., the Andean mountain range). This result is surprising, since the period considered is supposed to be synchronous with a sea-level rise (transitional period between Stages 8 and 7; Fig. 6), or high sea-level stand (Stage 7). The influence of them on the clay detrital output to the marine sedimentation should have enhanced the reworking of the downstream warmclimate soils depleted in these minerals. A possible explanation is that the sea-level rise or high sea-level stand was associated with a tectonic rejuvenation in the Andean range, balancing the reworking of soils and of rocky substrates, respectively. An argument for such a hypothesis is provided by the synchronous increase of the proportion of kaolinite and random mixed-layer minerals, two clay mineral groups dominantly formed in upstream soils and weathering profiles (Millot, 1964; Chamley, 1989). The relative increase of the kaolinite percentage toward the top of Zone $\mathrm{V}$ is attributed to a more humid climate contemporary to Stage 7 (Fig. 6) and enhanced continental hydrolysis processes.

\section{Clay Mineral Zone IV, Late Pleistocene}

The debris-flow deposits are marked by a fluctuating clay mineral composition associated with higher average amounts of smectite. Such an association is typical of a mixing of sediment sources on the fan and of active erosion processes occurring in the downstream areas 
of the Amazon river basin, where smectite was abundantly formed in the soils submitted to warm climate and restricted leaching. The clay mineral Zone IV could therefore reflect the development of regressive erosion that began in the downstream parts of the river basins when the sea level dropped. The fairly high amounts of random mixed layers $(14 c-14 s)$ in the zone also support the idea of active erosive mechanisms on exposed land masses, these minerals issuing dominantly from the lower levels of weathering profiles. The temporal variations registered in the clay mineral composition appear to result either from the erosion of different continental sources marked by different dominant soils sources or from the reworking of different areas in the proximal marine sedimentary environments. In addition, the subperiodic fluctuations of the smectite relative abundance and crystallinity (Fig. 7) suggest an almost cyclic stacking of the successive mass-transported sediments. The general upward decrease of the smectite abundance probably indicates the development of erosion processes, the preferentially downstream-forming clay mineral being progressively replaced by kaolinite ( $7 \AA / 10 \AA$ and $7 \AA / 17 \AA$ ratios), a mineral more easily formed in soils of the middle basin of the Amazon River.

\section{Clay Mineral Zone III, Late Pleistocene}

Poorly sampled, the subsequent clay mineral zone, which corresponds to the sandy formations of the HARP Channel-levee System 5 , expresses the continuation of active erosion and reworking processes synchronous with low sea level, and is responsible for the supply of detrital assemblages of fluctuating composition and variable origin.

\section{Clay Mineral Zone II, Late Pleistocene}

The sedimentation of the Channel-levee 5 deposits developed during the last glacial maximum and correlates with the highest influx of well-crystallized smectite (Fig. 7). This mineral is chiefly contained in the downstream soils of the Amazon River basins, and the relative increase in smectite could indicate drainage changes with more erosional processes of soils from the lower Amazon plains during the last low sea-level stand. The subcyclic variations of smectite supply could indicate the high disturbance potential of vegetation during the late Pleistocene expressed by palynological data (Absy et al., 1991; Pujos et al., 1995; Haberle, this volume). The lower part of Zone II, marked by maximum amounts of kaolinite before the Lake Mungo Excursion $(\sim 30 \mathrm{ka})$, probably indicates the start of this change with first the erosion of the more mature soils.

\section{Clay Mineral Zone I, Uppermost Pleistocene/Holocene}

The more recent stage marked by the maximum amounts of wellcrystallized smectite as well as by the lowest percentages of illite, chlorite, and random mixed layers, clearly points to the development of interglacial-type conditions enhancing the pedogenic processes (see Chamley, 1989). The soil-forming smectite is associated with high relative amounts of kaolinite in the uppermost level investigated, which supports the extension of pedogenic activity and soil erosion. Palynological data of this time interval in the same area indicate the development of the rain forest in the Amazon lowland basin and of the treeline in the upland basin (Haberle, this volume). Haberle underlines the increase of moisture and temperature as well as the disappearance of dry savanna species, factors favoring the formation of pedogenic kaolinite and the upstream trapping of rock-derived chlorite and illite.

\section{CONCLUSION}

The following conclusions can be drawn from this study:
1. The Amazon Fan was investigated from the clay mineral distribution in 16 Leg 155 sites drilled within a $260 \times 280 \mathrm{~km}$ area located off the river mouth in a water depth between 2750 and $4150 \mathrm{~m}$. The clay mineral assemblages determined from about 150 sediment samples encompass the middle Pleistocene to Holocene stages that are placed in a geographical, lithologic, and sedimentological context. The sediments are dominated by clay-rich facies associated with variable amounts of foraminifers, nannofossils, and thin ferric oxide-rich horizons.

2. The clay mineral assemblages are dominated by medium-crystalline smectite, illite, and kaolinite, associated with minor amounts of chlorite, random mixed layers (especially chloritesmectite), quartz, and feldspars. The relative abundance of the different clay species varies in space and time. All dominant clay minerals are considered to result from a terrigenous supply and express variations in the soil and rock sources due to paleoenvironmental changes. Smectite and kaolinite chiefly reflect the soil erosion in the lower and middle Amazon Basin, whereas illite, chlorite, and other clay-sized species dominantly reflect erosion processes occurring in the Andean range.

3. Sediments sampled between 0.1 and $5.5 \mathrm{mbsf}$ in all ODP sites display minor changes along a transect parallel to the main Amazon Channel. These changes reflect a moderate differential settling off the coast, expressed by a slight increase of smectite relative abundance. Such an increase is much more marked along an east-west transect normal to the coast, which reflects winnowing effects due to the action of the North Brazil Current. The more recent sediments are enriched in smectite and/or kaolinite, relative to the deposits located a few meters below the sea floor. This mineralogical change is independent of the lithology and chiefly attributed to a very recent increase in continental aridity and diminished erosional processes on adjacent land masses.

4. A high-resolution study of Holocene sediments at Hole 934A shows that the clay mineral successions are independent of the light/dark changes of the sediment color, pointing to the biogenic (white carbonate) and not terrigenous control of the recent cyclic changes expressed by the lithology. In addition, the occasional supply of illite and chlorite is attributed to the erosion of the Andean range at the end of the last glacial stage. The iron-bearing horizons extending widely in the Amazon Fan are relatively enriched in smectite.

5. The middle Pleistocene to Holocene successions expressed by Hole 931B, located on the western levee of the Channel 5 System, display fairly good correlations between the depositional units and the clay mineral zones that are interpreted as markers of paleoenvironmental changes. The relative abundance of illite in middle Pleistocene deposits is noteworthy. The debrisflow deposits characteristic of the lower part of the late Pleistocene display variable clay mineral assemblages that reflect the variety of submarine detrital sources associated with these reworked facies; the clay mineral associations of the debrisflow are dominated by smectite the abundance of which tends to slightly decrease uphole. Finally, the abundance of smectite-rich associations in the uppermost Pleistocene to Holocene sediments reflects both drainage changes during low sea-level stand and the recent sea-level rise favoring the erosion of the downstream soils of the Amazon River Basin, with accessory changes in the climate characteristics, namely the intensity of rainfall.

\section{ACKNOWLEDGMENTS}

We gratefully acknowledge R.D. Flood and two anonymous reviewers for their critical reviews and their suggestions, which greatly improved this paper. M. Bocquet and J. Carpentier were very helpful 
in conceiving the figures, and $\mathrm{Ph}$. Récourt efficiently carried out the XRD laboratory work. We warmly thank V. Kolla for his English edit. This research was supported by Institut National des Sciences de l’Univers grant, “Géosciences marines,” 1994/2.

\section{REFERENCES}

Absy, M.L., Cleef, A., Fournier, M., Martin, L., Servant, M., Siffedine, A., Ferriera da Silva, M., Soubies, S., Suguio, K., Turcq, B., and Van der Hammen, T., 1991. Mise en evidence de quatre phases d'ouverture de la forêt dense dans le sud-est de l'Amazonie au cours des 60000 dernières années. Première comparaison avec d'autres régions tropicales. C. $R$. Acad. Sci. Ser. 2, 312:673-678.

Allersma, E., 1971. Mud on the oceanic shelf off the Guyana. Symp. on Investigations of Resources in the Caribbean Sea and Adjacent Regions, 193-203.

Barreto, L.A., Milliman, J.D., Amaral, C.A.B., and Francisconi, O., 1975. Continental margin sedimentation off Brazil, Pt. II: Northern Brazil. Contrib. Sedimentol., 4:11-43.

Biscaye, P.E., 1965. Mineralogy and sedimentation of recent deep-sea clays in the Atlantic Ocean and adjacent seas and oceans. Geol. Soc. Am. Bull., 76:803-832.

Bouma, A.H., 1962. Sedimentology of Some Flysch Deposits: A Graphic Approach to Facies Interpretation: Amsterdam (Elsevier).

Bouquillon, A., 1987. Influence continentales et marines dans les sédiments Cénozoiques de l'ocean Indien Nord Oriental [thèse doct.]. Univ. Lille Flandres-Artois, France.

Bouquillon, A., and Debrabant, P., 1987. Distribution des minéraux argileux dans l'Océan Indien Nord Oriental. J. Rech. Oceanogr., 12:8-11.

Brindley, G.W., and Brown, G. (Eds.), 1980. Crystal Structures of Clay Minerals and Their X-ray Identification. Mineral. Soc. London Monogr., 5.

Chamley, H., 1989. Clay Sedimentology: Berlin (Springer-Verlag).

Damuth, J.E., 1977. Late Quaternary sedimentation in the western equatorial Atlantic. Geol. Soc. Am. Bull., 88:695-710.

Damuth, J.E., Flood, R.D., Knowsmann, R.O., Belderson, R.H., Gorini, M.A., 1988. Anatomy and growth patterns of Amazon deep-sea fan as revealed by long-range side-scan sonar (GLORIA) and high-resolution seismic studies. AAPG Bull., 72:885:911.

Damuth, J.E., Kowsmann, R.O., Flood, R.D., Belderson, R.H., and Gorini, M.A., 1983. Age relationships of distributary channels on Amazon deepsea fan: implications for fan growth pattern. Geology, 11:470-473.

Debrabant, P., Chamley, H., and Foulon, J., 1984. Paleoenvironmental implications of mineralogical and geochemical data in the Western Florida Straits (Leg 77, Deep Sea Drilling Project). In Buffler, R.T., Schlager, W., et al., Init. Repts. DSDP, 77: Washington (U.S. Govt. Printing Office), 377-396.

Debrabant, P., Krissek, L., Bouquillon, A., and Chamley, H., 1991. Clay mineralogy of neogene sediments of the western Arabian Sea: mineral abundances and paleoenvironmental implications. In Prell, W.L., Niitsuma, N., et al., Proc. ODP, Sci. Results, 117: College Station, TX (Ocean Drilling Program), 183-196.

Ericson, D.B., Ewing, M., Wollin, G., and Heezen, B.C., 1961. Atlantic deepsea sediment cores. Geol. Soc. Am. Bull., 72:193-286.

Ericson, D.B., and Wollin, G., 1968. Pleistocene climates and chronology in deep-sea sediments. Science, 162:1227-1234.

Fagel, N., 1994. Flux argileux du Néogène au Quaternaire dans l'Océan Indien Nord. Mise en évidence et interprétation. Soc. Geol. Nord, 22

Fagel, N., Debrabant, P., and André L., 1994. Clay supplies in the Central Indian Basin since the Late Miocene: climatic or tectonic control? Mar. Geol., 122:151-172.

Fagel, N., Debrabant, P., DeMenocal, P., and Demoulin, B., 1992. Utilisation des minéraux sédimentaires argileux pour la reconstitution des variations paléoclimatiques à court terme en Mer d'Arabie. Oceanol. Acta, 15:125137.

Flood, R.D., Manley, P.L., Kowsmann, R.O., Appi, C.J., and Pirmez, C., 1991. Seismic facies and late Quaternary growth of Amazon submarine fan. In Weimer, P., and Link, M.H. (Eds.), Seismic Facies and Sedimentary Processes of Submarine Fans and Turbidite Systems: New York (Springer), 415-433.

Geyer, W.R., Beardsley, R.C., Candela, J., Castro, B.M., Legeckis, R.V., Lentz, S.J., Limeburner, R., Miranda, L.B., and Trowbridge, J.H., 1991.The physical oceanography of the Amazon outflow. Oceanography, 4:8-14.
Gibbs, R.J., 1967. The geochemistry of the Amazon River system. Part I. The factors that control the salinity and the composition and concentration of the suspended solids. Geol. Soc. Am. Bull., 78:1203-1232.

, 1977. Clay mineral segregation in the marine environment. $J$. Sediment. Petrol., 47:237-243.

Guyot, J.L., 1992. Hydrogéochimie des fleuves de l'Amazonie Bolivienne. Paris (ORSTOM Edit).

Holtzapffel, T., 1985. Les minéraux argileux: préparation, analyse diffractométrique et détermination. Publ. Soc. Geol. Nord., 12.

Hoorn, C., 1994. An environmental reconstruction of the palaeo-Amazon River system (Middle-Late Miocene, NW Amazonia). Palaeogeogr., Palaeoclimatol., Palaeoecol., 112:187-238.

Hoorn, C., Guerrero, J., Sarmiento, G.A., and Lorente, M.A., 1995. Andean tectonics as a cause for changing drainage patterns in Miocene northern South America. Geology, 23:237-240.

Irion, G., 1984. Clay minerals of Amazonian soils. In Sioli, H. (Ed.), The Amazon Limnology and Landscape Ecology of a Mighty Tropical River and its Basin: Dordrecht (Junk), 537-579.

Johnsson, M.J., and Maede, R.H., 1990. Chemical weathering of fluvial sediments during alluvial storage: the Macuapanim island point bar, Solimoes river, Brazil. J. Sediment. Petrol., 60:827-842.

Lucas, J., 1962. La transformation des minéraux argileux dans la sédimentation: etudes sur les argiles du Trias. Mem. Serv. Carte Geol. Alsace-Lorraine, 23.

Manley, P.L., and Flood, R.D., 1988. Cyclic sediment deposition within Amazon deep-sea fan. AAPG Bull., 72:912-925.

Michalopoulos, P., and Aller, R.C., 1995. Rapid clay mineral formation in Amazon delta sediments: reverse weathering and oceanic elemental cycles. Science, 270: 614-617.

Milliman, J.D., Summerhayes, C.P., and Barretto, H.T., 1975. Quaternary sedimentation on the Amazon continental margin: a model. Geol. Soc. Am. Bull., 86:610-614.

Millot, G., 1964. Géologie des Argiles: Paris (Masson).

Moore, D.M., and Reynolds, R.C., Jr., 1989. X-ray Diffraction and the Identification and Analysis of Clay Minerals: Oxford (Oxford Univ. Press).

Nittrouer, C.A., Curtin, T.B., and DeMaster, D.J., 1986. Concentration and flux of suspended sediment on the Amazon continental shelf. Cont. Shelf Res., 6:151-174.

Nittrouer, C.A., DeMaster, D.J., Figueiredo, A.G., and Rine, J.M., 1991. AmasSeds: an interdisciplinary investigation of a complex coastal environment. Oceanography, 4:3-7.

Nittrouer, C.A., Kuehl, S.A., Sternberg, R.W., Figueiredo, A.G., Jr., Faria, L.E.C., 1995. An introduction to the geological significance of sediment transport and accumulation on the Amazon continental shelf. Mar. Geol., 125:177-192.

Patchineelam, S.M., 1993. Distribuçao espacial dos argilo minerais na plataforma continental amazonica [thesis]. Univ. Rio de Janeiro.

Pujos, M., Latouche, C., and Maillet, N., in press. Late Quaternary paleoceanography of the French Guiana continental shelf: clay-mineral evidences. Oceanologica Acta.

Robert, C., 1982. Modalité de la sédimentation argileuse en relation avec l'histoire géologique de l'Atlantique Sud [Ph.D. dissert.]. Univ. AixMarseille, France.

Shipboard Scientific Party, 1995a. Explanatory notes. In Flood, R.D., Piper, D.J.W., Klaus, A., et al., Proc. ODP, Init. Repts., 155: College Station, TX (Ocean Drilling Program), 47-81.

, 1995b. Site 934. In Flood, R.D., Piper, D.J.W., Klaus, A., et al., Proc. ODP, Init. Repts., 155: College Station, TX (Ocean Drilling Program), 241-272.

1995c. Site 931. In Flood, R.D., Piper, D.J.W., Klaus, A., et al., Proc. ODP, Init. Repts., 155: College Station, TX (Ocean Drilling Program), 123-164.

Stow, D.A.V, Howell, D.G., and Nelson, C.H., 1985. Sedimentary, tectonic, and sea level controls. In Bouma, A.H., Normark, W.R., and Barnes, N.E. (Eds.), Submarine Fans and Related Systems: New York (Springer), 1522.

Weaver, C.E., 1989. Clays, Muds, and Shales: New York (Elsevier), Dev. in Sedimentol. Ser., 44.

Date of initial receipt: 4 December 1995

Date of acceptance: 12 July 1996

Ms 155SR-227 